\title{
The Response to Arbitrarily Bandlimited Gaussian Noise of the Complex Stretch Processor Using a Conventional Range-Sidelobe-Reduction Window
}

\author{
John N. Spitzmiller \\ Simulation \& Integration Services, Parsons Government Services, Inc., Huntsville, AL, USA \\ Email: john.spitzmiller@parsons.com
}

How to cite this paper: Spitzmiller, J.N. (2018) The Response to Arbitrarily Bandlimited Gaussian Noise of the Complex Stretch Processor Using a Conventional Range-Sidelobe-Reduction Window. Journal of Signal and Information Processing, 9, 36-62.

https://doi.org/10.4236/jsip.2018.91003

Received: October 31, 2017

Accepted: February 9, 2018

Published: February 13, 2018

Copyright $\odot 2018$ by author and Scientific Research Publishing Inc. This work is licensed under the Creative Commons Attribution International License (CC BY 4.0).

http://creativecommons.org/licenses/by/4.0/

\begin{abstract}
This paper derives a mathematical description of the complex stretch processor's response to bandlimited Gaussian noise having arbitrary center frequency and bandwidth. The description of the complex stretch processor's random output comprises highly accurate closed-form approximations for the probability density function and the autocorrelation function. The solution supports the complex stretch processor's usage of any conventional rangesidelobe-reduction window. The paper then identifies two practical applications of the derived description. Digital-simulation results for the two identified applications, assuming the complex stretch processor uses the rectangular, Hamming, Blackman, or Kaiser window, verify the derivation's correctness through favorable comparison to the theoretically predicted behavior.
\end{abstract}

\section{Keywords}

Stretch Processing, Noise Jamming, Bandlimited Gaussian Noise, Range-Sidelobe-Reduction Windows

\section{Introduction}

Stretch processing [1]-[6] in radar uses relatively narrowband techniques to process wideband pulses with linear frequency modulation (LFM). Basic stretch processing [2] [3] (i.e., with no range-sidelobe-reduction window) yields the same fine range resolution and the same relatively high range-sidelobe levels produced by matched filtering. To reduce the range-sidelobe levels produced by basic stretch processing of LFM pulses, a practical stretch processor may apply a 
multiplicative window (e.g., a Hamming window) prior to the final Fourieranalysis stage [4] [5] [6].

Radar texts addressing noise in stretch processors [3] [4] typically consider only the case of broadband noise (e.g., receiver thermal noise). References [7] and [8] respectively characterized the response to bandlimited Gaussian noise (BLGN) having arbitrary center frequency and bandwidth of the complex stretch processor having no range-sidelobe-reduction window and the complex stretch processor employing a Hamming or Hann window. This paper extends the work in [7] [8] to characterize the output noise's probability density function (PDF) and autocorrelation function when the complex stretch processor uses any conventional multiplicative window to reduce the range-sidelobe levels. The output noise's PDF and autocorrelation function provide sufficient information for high-fidelity simulation of the complex stretch processor's output noise via standard techniques. Since the complex stretch processor is a linear system, a radar modeler may simply add the simulated noise to the complex stretch processor's simulated response to targets and clutter.

The derivation assumes the BLGN has arbitrary center frequency and bandwidth. Therefore, the results can describe the output noise due to input receiver thermal noise, broadband-noise jamming, spot-noise jamming, or even spectrally offset narrowband interference. The paper specifies a mathematical form for the window which can exactly represent the commonly used rectangular, Hamming, Hann, and Blackman windows and can closely approximate all other conventional windows.

Section 2 firstly specifies a simplified functional model of a radar employing a complex stretch processor with a range-sidelobe-reduction window. Section 2 then describes the processor's response to target-return signals. Section 3 derives a mathematical description, comprising the PDF and the autocorrelation function, of the complex stretch processor's theoretical response to arbitrarily bandlimited Gaussian noise. Section 4 presents simulation results which verify the derived expressions for two practical applications. Section 5 summarizes the technical approach, presents key findings, and suggests additional research.

\section{Review of Complex Stretch Processing}

This section reviews the fundamental operations of a radar using complex stretch processing. Figure 1 shows a simplified block diagram of the basic functional elements of a monostatic, pulsed radar employing complex stretch processing. This section's discussion uses the mathematical notation shown in Figure 1 which pictorially represents the complex stretch processor's stimulation by a target-return signal. For analytical convenience we assume the complex stretch processor comprises exclusively continuous-time (CT) subsystems.

\subsection{Transmitted Signal}

The radar's transmitter sends a single pulse, 


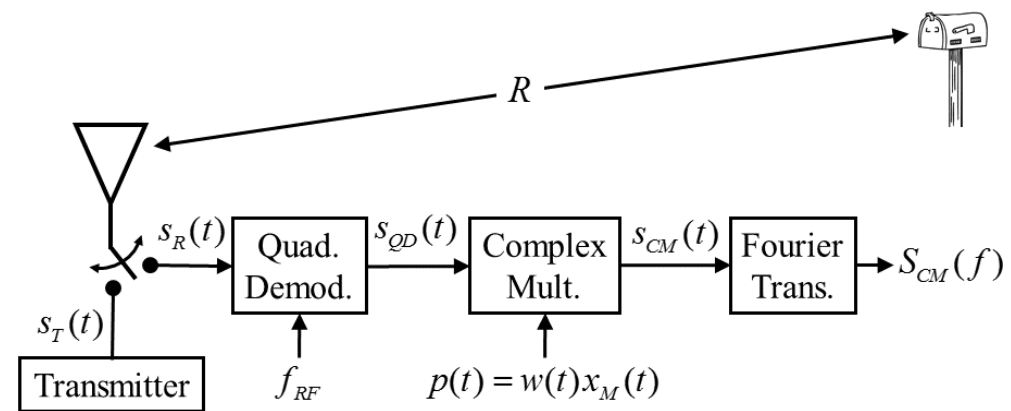

Figure 1. Block diagram of monostatic radar using complex stretch processing.

$$
s_{T}(t)=A_{T} \cos \left[2 \pi f_{R F} t+\Delta \phi_{i}(t)\right] \Pi\left(t / \tau_{p}\right),
$$

where $A_{T}$ is the pulse amplitude in volts, $f_{R F}$ is the center radio frequency (RF) in hertz, $t$ is time in seconds, $\tau_{p}$ is the pulse duration in seconds, and $\Delta \phi_{i}(t)$ is the instantaneous phase deviation in radians, to the transmit antenna. The transmit antenna radiates the pulse to a stationary point target at a slant range $R$ meters from the radar. In Equation (1)

$$
\Pi(x)= \begin{cases}1, & |x| \leq 1 / 2 \\ 0, & \text { otherwise }\end{cases}
$$

is the dimensionless unit-pulse function, and

$$
\Delta \phi_{i}(t)=2 \pi \int_{-\infty}^{t} \Delta f_{i}(\beta) \mathrm{d} \beta
$$

where, for an up-chirped LFM pulse with sweep bandwidth $B$ hertz,

$$
\Delta f_{i}(t)=\left(B / \tau_{p}\right) t \Pi\left(t / \tau_{p}\right)
$$

is the transmitted pulse's instantaneous frequency deviation in hertz. We substitute Equation (4) into Equation (3) and evaluate for Equation (1) to obtain

$$
s_{T}(t)=A_{T} \cos \left[2 \pi f_{R F} t+\pi B\left(t^{2}-\tau_{p}^{2} / 4\right) / \tau_{p}\right] \Pi\left(t / \tau_{p}\right) .
$$

\subsection{Received Signal}

The stationary point target instantaneously reradiates the incident pulse, so the receive antenna produces the voltage signal

$$
\begin{aligned}
& s_{R}(t)=\left(A_{R} / A_{T}\right) s_{T}\left(t-\tau_{d}\right) \\
& =A_{R} \cos \left\{2 \pi f_{R F} t-2 \pi f_{R F} \tau_{d}+\pi B\left[\left(t-\tau_{d}\right)^{2}-\tau_{p}^{2} / 4\right] / \tau_{p}\right\} \Pi\left[\left(t-\tau_{d}\right) / \tau_{p}\right] .
\end{aligned}
$$

In Equation (6)

$$
\tau_{d}=2 R / c
$$

is the round-trip propagation delay, and $c$ is the speed of light. The radar-range equation [9] determines the dimensionless ratio $A_{R} / A_{T}$.

\subsection{Quadrature Demodulator's Output}

Using reference frequency $f_{R F}$, the receive system's quadrature demodulator 
[10] produces the complex envelope

$$
\begin{aligned}
s_{Q D}(t) & =2 L P F\left[s_{R}(t) \cos \left(2 \pi f_{R F} t\right)\right]+j 2 L P F\left\{s_{R}(t)\left[-\sin \left(2 \pi f_{R F} t\right)\right]\right\} \\
& =A_{R} \mathrm{e}^{j\left\{-2 \pi f_{R F} \tau_{d}+\pi B t^{2} / \tau_{p}-2 \pi B \tau_{d} t / \tau_{p}+\pi B \tau_{d}^{2} / \tau_{p}-\pi B \tau_{p} / 4\right\}} \Pi\left[\left(t-\tau_{d}\right) / \tau_{p}\right] .
\end{aligned}
$$

In Equation (8) $\operatorname{LPF}(\cdot)$ indicates the operation of an ideal lowpass filter having a dimensionless passband gain of unity and a cutoff frequency between $B / 2$ and $2 f_{R F}-B / 2$. Thus, the quadrature demodulator's output has units of volts.

\subsection{Complex Multiplier's Output}

Assuming the stretch processor considers target slant ranges from $R_{\min }$ to $R_{\max }$, the slant ranges on this interval correspond to round-trip propagation delays from

$$
\tau_{\min }=2 R_{\min } / c
$$

to

$$
\tau_{\max }=2 R_{\max } / c .
$$

To support processing on slant ranges from $R_{\min }$ to $R_{\max }$, the complex multiplier of Figure 1 multiplies $s_{Q D}(t)$ with the dimensionless complex signal

$$
p(t)=w(t) x_{M}(t)
$$

where

$$
x_{M}(t)=\mathrm{e}^{j \Delta \phi_{M}(t)} \Pi\left[\left(t-\tau_{\text {avg }}\right) / T_{M}\right]
$$

is a complex heterodyne signal and $w(t)$ is a sidelobe-reduction window. In Equation (12)

$$
\begin{aligned}
\tau_{\text {avg }} & =\left(\tau_{\min }+\tau_{\max }\right) / 2, \\
T_{M} & =\tau_{\max }-\tau_{\min }+\tau_{p},
\end{aligned}
$$

and

$$
\Delta \phi_{M}(t)=\left(-\pi B t^{2}+2 \pi B \tau_{\text {avg }} t+\pi B T_{M}^{2} / 4-\pi B \tau_{\text {avg }}^{2}\right) / \tau_{p} .
$$

Note that Equation (15) is the instantaneous phase deviation corresponding to the instantaneous frequency deviation

$$
\Delta f_{M}(t)=\left(-B / \tau_{p}\right)\left(t-\tau_{\text {avg }}\right) \Pi\left[\left(t-\tau_{\text {avg }}\right) / T_{M}\right]
$$

which sweeps down through a bandwidth of

$$
B_{M}=B T_{M} / \tau_{p}>B
$$

Thus,

$$
x_{M}(t)=\mathrm{e}^{j\left(-\frac{\pi B}{\tau_{p}} t^{2}+\frac{2 \pi B \tau_{\text {avg }}}{\tau_{p}} t+\frac{\pi B T_{M}^{2}}{4 \tau_{p}}-\frac{\pi B \tau_{\text {avg }}^{2}}{\tau_{p}}\right)} \Pi\left[\left(t-\tau_{\text {avg }}\right) / T_{M}\right] .
$$

In Equation (11) $w(t)$ is identically zero outside $\tau_{\text {avg }}-T_{M} / 2 \leq t \leq \tau_{\text {avg }}+T_{M} / 2$. Mathematically, 


$$
w(t)=w_{p}(t) \Pi\left[\left(t-\tau_{\text {avg }}\right) / T_{M}\right],
$$

where

$$
w_{p}(t)=w(t), \tau_{\text {avg }}-T_{M} / 2 \leq t \leq \tau_{\text {avg }}+T_{M} / 2
$$

and $w_{p}(t)$ can have any form outside $\tau_{\text {avg }}-T_{M} / 2 \leq t \leq \tau_{\text {avg }}+T_{M} / 2$.

Equation (18) and Equation (19) have the common time-limiting factor $\Pi\left[\left(t-\tau_{\text {avg }}\right) / T_{M}\right]$ whose nonzero portion always fully overlaps the nonzero portion of $\Pi\left[\left(t-\tau_{d}\right) / \tau_{p}\right]$ in Equation (8). We can therefore express the complex multiplier's output voltage signal as

$$
\begin{aligned}
s_{C M}(t) & =p(t) s_{Q D}(t)=w_{p}(t) x_{M}(t) s_{Q D}(t) \\
& =A_{R} \mathrm{e}^{j\left[2 \pi B\left(\tau_{\text {avg }}-\tau_{d}\right) t / \tau_{p}+\theta\right]} \Pi\left[\left(t-\tau_{d}\right) / \tau_{p}\right] w_{p}(t),
\end{aligned}
$$

where

$$
\theta=\frac{\pi B T_{M}^{2}}{4 \tau_{p}}-\frac{\pi B\left(\tau_{a v g}^{2}-\tau_{d}^{2}\right)}{\tau_{p}}-2 \pi f_{R F} \tau_{d}-\frac{\pi B \tau_{p}}{4}
$$

Assuming

$$
W_{p}(f)=\int_{-\infty}^{\infty} w_{p}(t) \mathrm{e}^{-j 2 \pi f t} \mathrm{~d} t
$$

exists, the Fourier transform of Equation (21), having units of volt-seconds or volts/hertz, is

$$
\begin{aligned}
& S_{C M}(f) \\
& =A_{R} \tau_{p} \mathrm{e}^{j \theta} W_{p}(f) * \mathrm{e}^{-j 2 \pi\left[f-B\left(\tau_{\text {avg }}-\tau_{d}\right) / \tau_{p}\right] \tau_{d}} \operatorname{sinc}\left\{\tau_{p}\left[f-B\left(\tau_{\text {avg }}-\tau_{d}\right) / \tau_{p}\right]\right\},
\end{aligned}
$$

where * in Equation (24) denotes linear convolution. Thus, we desire a mathematical form for $w_{p}(t)$ which equals $w(t)$ on $\tau_{\text {avg }}-T_{M} / 2 \leq t \leq \tau_{\text {avg }}+T_{M} / 2$ and has a convenient Fourier transform. The periodic extension of $w(t)$ outside $\tau_{\text {avg }}-T_{M} / 2 \leq t \leq \tau_{\text {avg }}+T_{M} / 2$ satisfies these two criteria. Mathematically,

$$
w_{p}(t)=\sum_{k=-\infty}^{\infty} w\left(t-k T_{M}\right)
$$

Since this $w_{p}(t)$ is periodic with period $T_{M}$, we can express it as the Fourier series

$$
w_{p}(t)=\sum_{n=-\infty}^{\infty} W_{p}[n] \mathrm{e}^{j 2 \pi\left(n / T_{M}\right) t},
$$

where the Fourier series' coefficients are

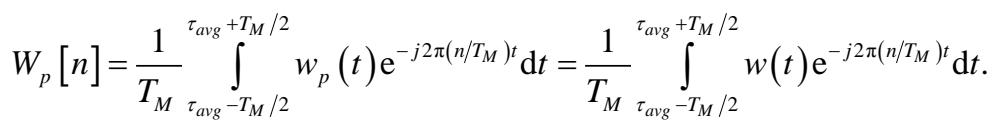

Since $\Pi\left[\left(t-\tau_{\text {avg }}\right) / T_{M}\right]$ temporally limits $w(t)$, we can also express Equation (27) as

$$
W_{p}[n]=\frac{1}{T_{M}} \int_{-\infty}^{\infty} w(t) \mathrm{e}^{-j 2 \pi\left(n / T_{M}\right) t} \mathrm{~d} t=\frac{1}{T_{M}} W\left(n / T_{M}\right)
$$


where

$$
W(f)=\int_{-\infty}^{\infty} w(t) \mathrm{e}^{-j 2 \pi f t} \mathrm{~d} t .
$$

The Fourier transform of Equation (26) is

$$
W_{p}(f)=\sum_{n=-\infty}^{\infty} W_{p}[n] \delta\left(f-n / T_{M}\right),
$$

where $\delta$ denotes the continuous-variable Dirac delta (impulse) function.

Substituting Equation (30) into Equation (24) gives us

$$
\begin{aligned}
S_{C M}(f)= & A_{R} \tau_{p} \mathrm{e}^{j \theta}\left\{\sum_{n=-\infty}^{\infty} W_{p}[n] \delta\left(f-n / T_{M}\right)\right\} \\
& * \mathrm{e}^{-j 2 \pi\left[f-B\left(\tau_{\text {avg }}-\tau_{d}\right) / \tau_{p}\right] \tau_{d}} \operatorname{sinc}\left\{\tau_{p}\left[f-B\left(\tau_{\text {avg }}-\tau_{d}\right) / \tau_{p}\right]\right\} \\
= & A_{R} \tau_{p} \mathrm{e}^{j \theta} \sum_{n=-\infty}^{\infty} W_{p}[n] \mathrm{e}^{-j 2 \pi\left[f-\frac{n}{T_{M}}-\frac{B\left(\tau_{\text {avg }}-\tau_{d}\right)}{\tau_{p}}\right] \tau_{d}} \\
& \times \operatorname{sinc}\left\{\tau_{p}\left[f-\frac{n}{T_{M}}-\frac{B\left(\tau_{a v g}-\tau_{d}\right)}{\tau_{p}}\right]\right\},
\end{aligned}
$$

where

$$
\operatorname{sinc}(x)=\sin (\pi x) /(\pi x) .
$$

For any conventional window, the peak magnitude of Equation (31) occurs either exactly or very nearly at frequency

$$
f_{\text {peak }}=B\left(\tau_{\text {avg }}-\tau_{d}\right) / \tau_{p},
$$

which maps to slant range

$$
R_{\text {peak }}=(c / 2)\left(-\tau_{p} f_{\text {peak }} / B+\tau_{\text {avg }}\right)=(c / 2) \tau_{d}=R .
$$

The slant-range interval $R_{\min } \leq R \leq R_{\max }$ maps to the frequency interval $f\left(R_{\text {max }}\right) \leq f \leq f\left(R_{\text {min }}\right)$, where

$$
f\left(R_{\text {max }}\right)=B\left(\tau_{\text {avg }}-\tau_{\max }\right) / \tau_{p}<0
$$

and

$$
f\left(R_{\min }\right)=B\left(\tau_{\text {avg }}-\tau_{\min }\right) / \tau_{p}=-f\left(R_{\max }\right)>0
$$

\section{Complex Stretch Processor's Theoretical Response to BLGN}

This section mathematically characterizes the complex stretch processor's theoretical response to BLGN having arbitrary bandwidth and center frequency. We firstly describe the BLGN. We then determine the PDF and autocorrelation function of the receive system's response to the BLGN. Specifically, we show the complex stretch processor's output is complex, zero mean, and Gaussian with independent real and imaginary parts. We then derive the autocorrelation function of the complex stretch processor's output. From the autocorrelation function, we find the variance to complete the PDF's description. This section's discussion uses the mathematical notation shown in Figure 2 which pictorially 


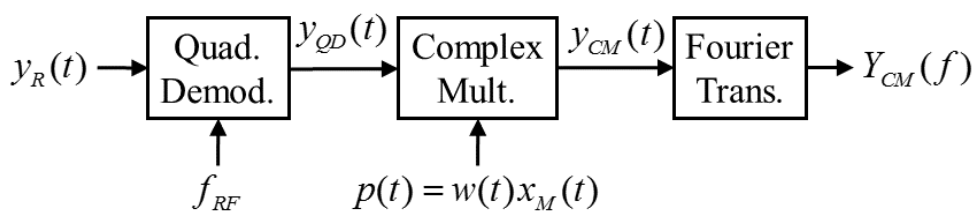

Figure 2. Complex stretch processor stimulated by arbitrarily bandlimited Gaussian noise.

represents the complex stretch processor's stimulation by arbitrarily bandlimited Gaussian noise.

\subsection{BLGN Description}

The BLGN at the complex stretch processor's input is a real random-voltage signal having mathematical form

$$
y_{R}(t)=y_{I}(t) \cos \left(2 \pi f_{y} t\right)-y_{Q}(t) \sin \left(2 \pi f_{y} t\right),
$$

where $f_{y}$ is the BLGN's center RF. As given in [11], $y_{I}(t)$ and $y_{Q}(t)$ are real, independent, lowpass, zero-mean, Gaussian, wide-sense-stationary (WSS) random signals having common power spectral density (PSD)

$$
S_{y_{I}}(f)=S_{y_{Q}}(f)=N_{y} \Pi\left(f / B_{y}\right),
$$

where $B_{y}$ is the BLGN's RF bandwidth. Since $y_{R}(t)$ is a voltage signal, Equation (38) and $N_{y}$ have units of volts ${ }^{2} /$ hertz. We assume $f_{y} \gg B_{y}$, so $y_{R}(t)$ is a narrowband, zero-mean, Gaussian, WSS random signal having PSD

$$
S_{y_{R}}(f)=\frac{1}{2} N_{y}\left\{\Pi\left[\left(f-f_{y}\right) / B_{y}\right]+\Pi\left[\left(f+f_{y}\right) / B_{y}\right]\right\}
$$

as depicted in Figure 3.

\subsection{Quadrature Demodulator's Output}

The quadrature demodulator applies the mathematical action of Equation (8) to $y_{R}(t)$ to produce the complex random-voltage signal

$$
\begin{aligned}
y_{Q D}(t)= & y_{I}(t) \cos \left[2 \pi\left(f_{y}-f_{R F}\right) t\right]-y_{Q}(t) \sin \left[2 \pi\left(f_{y}-f_{R F}\right) t\right] \\
& +j\left\{y_{I}(t) \sin \left[2 \pi\left(f_{y}-f_{R F}\right) t\right]+y_{Q}(t) \cos \left[2 \pi\left(f_{y}-f_{R F}\right) t\right]\right\} .
\end{aligned}
$$

Since $y_{I}(t)$ and $y_{Q}(t)$ are zero mean and Gaussian, $y_{Q D}(t)$ is also zero mean and Gaussian [12]. Straightforward analysis of Equation (40) establishes the fact that $y_{Q D}(t)$ is WSS with PSD

$$
S_{y_{Q D}}(f)=2 N_{y} \Pi\left\{\left[f-\left(f_{y}-f_{R F}\right)\right] / B_{y}\right\} .
$$

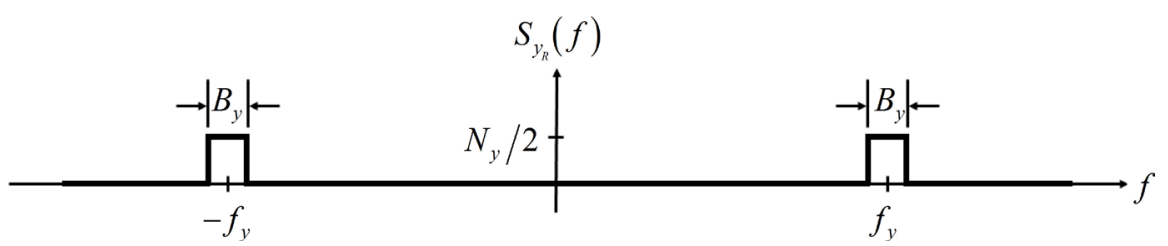

Figure 3. Power spectral density of arbitrarily bandlimited Gaussian noise. 


\subsection{Fourier Transform's Output}

The Fourier transform of the complex multiplier's output

$$
y_{C M}(t)=p(t) y_{Q D}(t)
$$

is

$$
Y_{C M}(f)=\int_{-\infty}^{\infty} y_{C M}(t) \mathrm{e}^{-j 2 \pi f t} \mathrm{~d} t=\int_{-\infty}^{\infty} p(t) y_{Q D}(t) \mathrm{e}^{-j 2 \pi f t} \mathrm{~d} t .
$$

Since $y_{Q D}(t)$ is a time-domain random process having units of volts, $Y_{C M}(f)$ is a frequency-domain random process having units of volt-seconds or volts/ hertz. Straightforward but tedious mathematics show the real and imaginary parts of $Y_{C M}(f)$ to be uncorrelated and to have equal variances. For a specific value of $f$ (say, $\left.f_{1}\right), Y_{C M}(f)$ is the complex Gaussian random variable (RV) [12]

$$
Y_{C M}\left(f_{1}\right)=\int_{-\infty}^{\infty} y_{C M}(t) \mathrm{e}^{-j 2 \pi f_{1} t} \mathrm{~d} t=\int_{-\infty}^{\infty} p(t) y_{Q D}(t) \mathrm{e}^{-j 2 \pi f_{1} t} \mathrm{~d} t .
$$

Since the real and imaginary parts of $Y_{C M}\left(f_{1}\right)$ are uncorrelated and Gaussian RVs, the RVs are also independent. Since $Y_{C M}\left(f_{1}\right)$ is a complex Gaussian RV, the mean, correlation, and variance of its real and imaginary parts completely specify the complex RV's PDF (i.e., the joint PDF of the RV's real and imaginary parts [13]). The RV has mean

$$
E\left[Y_{C M}\left(f_{1}\right)\right]=\int_{-\infty}^{\infty} p(t) E\left[y_{Q D}(t)\right] \mathrm{e}^{-j 2 \pi f_{1} t} \mathrm{~d} t=0 \forall f_{1},
$$

where $E(Z)$ denotes the expected value of the generally complex RV $Z$. Thus, the mean of both the real and imaginary parts of $Y_{C M}\left(f_{1}\right)$ is zero. Since the real and imaginary parts are independent and zero mean, their correlation is zero. We find the variance of the RV's real and imaginary parts by finding the autocorrelation function of $Y_{C M}(f)$, setting both frequency arguments equal to $f_{1}$, and dividing the result by two.

The autocorrelation function of $Y_{C M}(f)$ is

$$
\begin{aligned}
R_{Y_{C M}}\left(f_{1}, f_{2}\right) & =E\left[Y_{C M}\left(f_{1}\right) Y_{C M}^{*}\left(f_{2}\right)\right] \\
& =E\left[\int_{-\infty}^{\infty} p(t) y_{Q D}(t) \mathrm{e}^{-j 2 \pi f_{1} t} \mathrm{~d} t \int_{-\infty}^{\infty} p^{*}(\gamma) y_{Q D}^{*}(\gamma) \mathrm{e}^{j 2 \pi f_{2} \gamma} \mathrm{d} \gamma\right] \\
& =\int_{-\infty}^{\infty} \int_{-\infty}^{\infty} p(t) E\left[y_{Q D}(t) y_{Q D}^{*}(\gamma)\right] \mathrm{e}^{-j 2 \pi f_{1} t} p^{*}(\gamma) \mathrm{e}^{j 2 \pi f_{2} \gamma} \mathrm{d} \gamma \mathrm{d} t \\
& =\int_{-\infty}^{\infty} \int_{-\infty}^{\infty} p(t) R_{y_{Q D}}(t-\gamma) \mathrm{e}^{-j 2 \pi f_{1} t} p^{*}(\gamma) \mathrm{e}^{j 2 \pi f_{2} \gamma} \mathrm{d} \gamma \mathrm{d} t .
\end{aligned}
$$

Since $y_{Q D}(t)$ is WSS, its autocorrelation function is the inverse Fourier transform of its PSD, so

$$
R_{y_{Q D}}(t-\gamma)=\int_{-\infty}^{\infty} S_{y_{Q D}}(f) \mathrm{e}^{j 2 \pi f(t-\gamma)} \mathrm{d} f .
$$

Therefore, we can write Equation (46) as 


$$
\begin{aligned}
& R_{Y_{C M}}\left(f_{1}, f_{2}\right) \\
& =\int_{-\infty}^{\infty} \int_{-\infty}^{\infty} p(t)\left[\int_{-\infty}^{\infty} S_{y_{Q D}}(f) \mathrm{e}^{j 2 \pi f(t-\gamma)} \mathrm{d} f\right] \mathrm{e}^{-j 2 \pi f_{1} t} p^{*}(\gamma) \mathrm{e}^{j 2 \pi f_{2} \gamma} \mathrm{d} \gamma \mathrm{d} t \\
& =\int_{-\infty}^{\infty} S_{y_{Q D}}(f)\left[\int_{-\infty}^{\infty} p(t) \mathrm{e}^{-j 2 \pi f_{1} t} \mathrm{e}^{j 2 \pi f t} \mathrm{~d} t\right]\left[\int_{-\infty}^{\infty} p^{*}(\gamma) \mathrm{e}^{j 2 \pi f_{2} \gamma} \mathrm{e}^{-j 2 \pi f \gamma} \mathrm{d} \gamma\right] \mathrm{d} f \\
& =\int_{-\infty}^{\infty} S_{y_{Q D}}(f) P\left(f_{1}-f\right) P^{*}\left(f_{2}-f\right) \mathrm{d} f,
\end{aligned}
$$

where

$$
\begin{aligned}
P(f) & =\int_{-\infty}^{\infty} p(t) \mathrm{e}^{-j 2 \pi f t} \mathrm{~d} t=\int_{-\infty}^{\infty} w(t) x_{M}(t) \mathrm{e}^{-j 2 \pi f t} \mathrm{~d} t \\
& =\int_{-\infty}^{\infty} w_{p}(t) X_{M}(t) \mathrm{e}^{-j 2 \pi f t} \mathrm{~d} t=W_{p}(f) * X_{M}(f) \\
& =\int_{-\infty}^{\infty} W_{p}(\beta) X_{M}(f-\beta) \mathrm{d} \beta .
\end{aligned}
$$

Substituting Equation (30) into Equation (49) gives us

$$
\begin{aligned}
P(f) & =\int_{-\infty}^{\infty} \sum_{n=-\infty}^{\infty} W_{p}[n] \delta\left(\beta-n / T_{M}\right) X_{M}(f-\beta) \mathrm{d} \beta \\
& =\sum_{n=-\infty}^{\infty} W_{p}[n] \int_{-\infty}^{\infty} \delta\left(\beta-n / T_{M}\right) X_{M}(f-\beta) \mathrm{d} \beta \\
& =\sum_{n=-\infty}^{\infty} W_{p}[n] X_{M}\left(f-n / T_{M}\right) .
\end{aligned}
$$

In a practical stretch processor, the heterodyne signal's time-bandwidth product $B_{M} T_{M}$ very greatly exceeds unity, so [7]

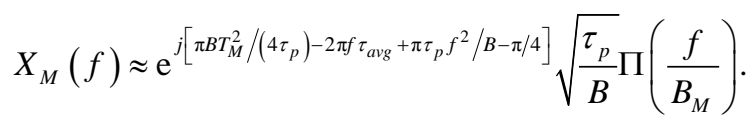

Substituting Equation (51) into Equation (50) gives (after simplification)

$$
\begin{aligned}
& P(f) \\
& \left.\approx \sum_{n=-\infty}^{\infty} W_{p}[n] \mathrm{e}^{j\left[\pi B T_{M}^{2} /\left(4 \tau_{p}\right)-2 \pi\left(f-n / T_{M}\right) \tau_{\text {vvg }}+\pi \tau_{p}\left(f-n / T_{M}\right)^{2} / B-\pi / 4\right.}\right] \sqrt{\frac{\tau_{p}}{B}} \Pi\left(\frac{f-n / T_{M}}{B_{M}}\right) .
\end{aligned}
$$

All conventional windows have energy spectral densities concentrated around $f=0 \quad[14]$, so

$$
W_{p}[n] \approx 0,|n|>N_{W},
$$

for some positive integer $N_{W}$. Therefore, we can make the further approximation

$$
\begin{aligned}
& P(f) \\
& \left.\approx \sum_{n=-N_{W}}^{N_{W}} W_{p}[n] \mathrm{e}^{j\left[\pi B T_{M}^{2} /\left(4 \tau_{p}\right)-2 \pi\left(f-n / T_{M}\right) \tau_{\alpha v g}+\pi \tau_{p}\left(f-n / T_{M}\right)^{2} / B-\pi / 4\right.}\right] \sqrt{\frac{\tau_{p}}{B}} \Pi\left(\frac{f-n / T_{M}}{B_{M}}\right) .
\end{aligned}
$$

For practical stretch processors, the sweep bandwidth $B_{M}$ very greatly ex- 
ceeds $1 / T_{M}$, so

$$
\Pi\left[\left(f-n / T_{M}\right) / B_{M}\right] \approx \Pi\left(f / B_{M}\right),-N_{W} \leq n \leq N_{W},
$$

assuming

$$
N_{W} / T_{M} \ll B_{M} .
$$

Equation (55) and Equation (56) permit the further approximation

$$
P(f) \approx \sqrt{\frac{\tau_{p}}{B}} \Pi\left(\frac{f}{B_{M}}\right) \sum_{n=-N_{W}}^{N_{W}} W_{p}[n] \mathrm{e}^{j\left[\pi B T_{M}^{2} /\left(4 \tau_{p}\right)-2 \pi\left(f-n / T_{M}\right) \tau_{\text {vvg }}+\pi \tau_{p}\left(f-n / T_{M}\right)^{2} / B-\pi / 4\right]} .
$$

From Equation (57) we immediately obtain

$$
\begin{aligned}
& P\left(f_{1}-f\right) \\
& \approx \sqrt{\frac{\tau_{p}}{B}} \Pi\left(\frac{f_{1}-f}{B_{M}}\right) \sum_{n=-N_{W}}^{N_{W}} W_{p}[n] \mathrm{e}^{j\left[\pi B T_{M}^{2} /\left(4 \tau_{p}\right)-2 \pi\left(f_{1}-f-n / T_{M}\right) \tau_{\text {avg }}+\pi \tau_{p}\left(f_{1}-f-n / T_{M}\right)^{2} / B-\pi / 4\right]}
\end{aligned}
$$

and

$$
\begin{aligned}
& P^{*}\left(f_{2}-f\right) \\
& \approx \sqrt{\frac{\tau_{p}}{B}} \Pi\left(\frac{f_{2}-f}{B_{M}}\right) \sum_{n=-N_{W}}^{N_{W}} W_{p}^{*}[n] \mathrm{e}^{-j\left[\pi B T_{M}^{2} /\left(4 \tau_{p}\right)-2 \pi\left(f_{2}-f-n / T_{M}\right) \tau_{\alpha v g}+\pi \tau_{p}\left(f_{2}-f-n / T_{M}\right)^{2} / B-\pi / 4\right]} .
\end{aligned}
$$

Substituting Equation (41), Equation (58), and Equation (59) into Equation (48) gives us

$$
\begin{aligned}
& R_{\mathrm{Y}_{C M}}\left(f_{1}, f_{2}\right) \approx \frac{2 N_{y} \tau_{p}}{B} \int_{-\infty}^{\infty} \Pi\left[\frac{f-\left(f_{y}-f_{R F}\right)}{B_{y}}\right] \Pi\left(\frac{f_{1}-f}{B_{M}}\right) \Pi\left(\frac{f_{2}-f}{B_{M}}\right) \\
& \left.\times\left\{\sum_{n=-N_{W}}^{N_{W}} W_{p}[n] \mathrm{e}^{j\left[\pi B T_{M}^{2} /\left(4 \tau_{p}\right)-2 \pi\left(f_{1}-f-n / T_{M}\right) \tau_{\text {avg }}+\pi \tau_{p}\left(f_{1}-f-n / T_{M}\right)^{2} / B-\pi / 4\right.}\right]\right\} \\
& \times\left\{\sum_{m=-N_{W}}^{N_{W}} W_{p}^{*}[m] \mathrm{e}^{-j\left[\pi B T_{M}^{2} /\left(4 \tau_{p}\right)-2 \pi\left(f_{2}-f-m / T_{M}\right) \tau_{\text {avg }}+\pi \tau_{p}\left(f_{2}-f-m / T_{M}\right)^{2} / B-\pi / 4\right.}\right] \mathrm{d} f \\
& =\frac{2 N_{y} \tau_{p}}{B} \int_{-\infty}^{\infty} \Pi\left[\frac{f-\left(f_{y}-f_{R F}\right)}{B_{y}}\right] \Pi\left(\frac{f_{1}-f}{B_{M}}\right) \Pi\left(\frac{f_{2}-f}{B_{M}}\right) \\
& \times \sum_{n=-N_{W}}^{N_{W}} \sum_{m=-N_{W}}^{N_{W}} W_{p}[n] W_{p}^{*}[m] \mathrm{e}^{j\left[-2 \pi\left(f_{1}-f-n / T_{M}\right) \tau_{\text {avg }}+\pi \tau_{p}\left(f_{1}-f-n / T_{M}\right)^{2} / B\right]} \\
& \times \mathrm{e}^{-j\left[-2 \pi\left(f_{2}-f-m / T_{M}\right) \tau_{\text {avg }}+\pi \tau_{p}\left(f_{2}-f-m / T_{M}\right)^{2} / B\right]} \mathrm{d} f \\
& =\frac{2 N_{y} \tau_{p}}{B} \mathrm{e}^{-j 2 \pi\left(f_{1}-f_{2}\right) \tau_{\text {vvg }}} \mathrm{e}^{j \pi \tau_{p}\left(f_{1}^{2}-f_{2}^{2}\right) / B} \\
& \times \sum_{n=-N_{W}}^{N_{W}} \sum_{m=-N_{W}}^{N_{W}} W_{p}[n] W_{p}^{*}[m] \mathrm{e}^{j 2 \pi(n-m) \tau_{\text {avg }} / T_{M}} \mathrm{e}^{j \pi \tau_{p}\left(n^{2}-m^{2}\right) /\left(B T_{M}^{2}\right)} \\
& \times \mathrm{e}^{-j 2 \pi \tau_{p}\left(n f_{1}-m f_{2}\right) /\left(B T_{M}\right)} \int_{-\infty}^{\infty} \mathrm{e}^{j 2 \pi \tau_{p}(n-m) f /\left(B T_{M}\right)} \mathrm{e}^{-j 2 \pi \tau_{p}\left(f_{1}-f_{2}\right) f / B} \\
& \times \Pi\left[\frac{f-\left(f_{y}-f_{R F}\right)}{B_{y}}\right] \Pi\left(\frac{f_{1}-f}{B_{M}}\right) \Pi\left(\frac{f_{2}-f}{B_{M}}\right) \mathrm{d} f .
\end{aligned}
$$


The variance of the output noise at frequency $f_{1}$ is

$$
\begin{aligned}
& \operatorname{var}\left[Y_{C M}\left(f_{1}\right)\right]=R_{Y_{C M}}\left(f_{1}, f_{1}\right) \\
& \approx \frac{2 N_{y} \tau_{p}}{B} \sum_{n=-N_{W}}^{N_{W}} \sum_{m=-N_{W}}^{N_{W}} W_{p}[n] W_{p}^{*}[m] \mathrm{e}^{j 2 \pi(n-m) \tau_{\text {avg }} / T_{M}} \mathrm{e}^{j \pi \tau_{p}\left(n^{2}-m^{2}\right) /\left(B T_{M}^{2}\right)} \\
& \times \mathrm{e}^{-j 2 \pi \tau_{p} f_{1}(n-m) /\left(B T_{M}\right)} \int_{-\infty}^{\infty} \mathrm{e}^{j 2 \pi \tau_{p}(n-m) f /\left(B T_{M}\right)} \Pi\left[\frac{f-\left(f_{y}-f_{R F}\right)}{B_{y}}\right] \Pi\left(\frac{f_{1}-f}{B_{M}}\right) \mathrm{d} f .
\end{aligned}
$$

For values of $f_{1}$ outside the frequency interval

$$
I_{1}=\left[f_{y}-f_{R F}-\frac{B_{y}}{2}-\frac{B_{M}}{2}, f_{y}-f_{R F}+\frac{B_{y}}{2}+\frac{B_{M}}{2}\right],
$$

the two $\Pi$ functions in the integrand of Equation (61) have no nonzero overlap, so the output-noise variance is zero, meaning the BLGN does not corrupt the Fourier transform's output at frequencies outside $I_{1}$. Since the stretch processor only considers frequencies on $\left[f\left(R_{\max }\right), f\left(R_{\min }\right)\right]$, the BLGN only corrupts the stretch processor's output from

$$
f_{a}=\max \left[f_{y}-f_{R F}-B_{y} / 2-B_{M} / 2, f\left(R_{\max }\right)\right]
$$

to

$$
f_{b}=\min \left[f_{y}-f_{R F}+B_{y} / 2+B_{M} / 2, f\left(R_{\min }\right)\right] .
$$

Now, we respectively define

$$
f_{l}\left(f_{1}, f_{2}\right)=\max \left[f_{y}-f_{R F}-\frac{B_{y}}{2}, f_{1}-\frac{B_{M}}{2}, f_{2}-\frac{B_{M}}{2}\right]
$$

and

$$
f_{u}\left(f_{1}, f_{2}\right)=\min \left[f_{y}-f_{R F}+\frac{B_{y}}{2}, f_{1}+\frac{B_{M}}{2}, f_{2}+\frac{B_{M}}{2}\right]
$$

as the lower and upper frequency boundaries of the nonzero overlap of the three $\Pi$ functions in the integrand of Equation (60). Note: If $f_{l}\left(f_{1}, f_{2}\right)>f_{u}\left(f_{1}, f_{2}\right)$, the product of the three $\Pi$ functions is zero for all $f$, so Equation (60) is practically zero for all $\left(f_{1}, f_{2}\right)$ such that $f_{l}\left(f_{1}, f_{2}\right)$ exceeds $f_{u}\left(f_{1}, f_{2}\right)$. Assuming values of $\left(f_{1}, f_{2}\right)$ such that $f_{l}\left(f_{1}, f_{2}\right)<f_{u}\left(f_{1}, f_{2}\right)$, we determine the autocorrelation function to be

$$
\begin{aligned}
R_{Y_{C M}}\left(f_{1}, f_{2}\right) \approx & \frac{2 N_{y} \tau_{p}}{B} \mathrm{e}^{-j 2 \pi\left(f_{1}-f_{2}\right) \tau_{\text {avg }}} \mathrm{e}^{j \pi \tau_{p}\left(f_{1}^{2}-f_{2}^{2}\right) / B} \\
& \times \sum_{n=-N_{W}}^{N_{W}} \sum_{m=-N_{W}}^{N_{W}} W_{p}[n] W_{p}^{*}[m] \mathrm{e}^{j 2 \pi(n-m) \tau_{\text {avg }} / T_{M}} \\
& \times \mathrm{e}^{j \pi \tau_{p}\left(n^{2}-m^{2}\right) /\left(B T_{M}^{2}\right)} \mathrm{e}^{-j 2 \pi \tau_{p}\left(n f_{1}-m f_{2}\right) /\left(B T_{M}\right)} \\
& \times \int_{-\infty}^{\infty} \mathrm{e}^{-j 2 \pi \frac{\tau_{p}}{B}\left(f_{1}-f_{2}-\frac{n-m}{T_{M}}\right) f} \Pi\left[\frac{f-f_{c}\left(f_{1}, f_{2}\right)}{B_{e q}\left(f_{1}, f_{2}\right)}\right] \mathrm{d} f .
\end{aligned}
$$

In Equation (67) 


$$
f_{c}\left(f_{1}, f_{2}\right)=\left[f_{l}\left(f_{1}, f_{2}\right)+f_{u}\left(f_{1}, f_{2}\right)\right] / 2
$$

and

$$
B_{e q}\left(f_{1}, f_{2}\right)=f_{u}\left(f_{1}, f_{2}\right)-f_{l}\left(f_{1}, f_{2}\right)
$$

respectively represent the center frequency and spectral width of the three $\Pi$ functions' nonzero product. Finally, we evaluate the integral in Equation (67) to obtain

$$
\begin{aligned}
& R_{Y_{C M}}\left(f_{1}, f_{2}\right) \approx \frac{2 N_{y} \tau_{p} B_{e q}\left(f_{1}, f_{2}\right)}{B} \mathrm{e}^{-j 2 \pi\left(f_{1}-f_{2}\right) \tau_{\text {vvg }}} \mathrm{e}^{j \pi \tau_{p}\left(f_{1}^{2}-f_{2}^{2}\right) / B} \\
& \times \sum_{n=-N_{W}}^{N_{W}} \sum_{m=-N_{W}}^{N_{W}} W_{p}[n] W_{p}^{*}[m] \mathrm{e}^{j 2 \pi(n-m) \tau_{a v g} / T_{M}} \mathrm{e}^{j \pi \tau_{p}\left(n^{2}-m^{2}\right) /\left(B T_{M}^{2}\right)} \\
& \times \mathrm{e}^{-j 2 \pi \tau_{p}\left(n f_{1}-m f_{2}\right) /\left(B T_{M}\right)} \mathrm{e}^{-j 2 \pi \frac{\tau_{p}}{B}\left(f_{1}-f_{2}-\frac{n-m}{T_{M}}\right) f_{c}\left(f_{1}, f_{2}\right)} \\
& \times \operatorname{sinc}\left[B_{e q}\left(f_{1}, f_{2}\right) \frac{\tau_{p}}{B}\left(f_{1}-f_{2}-\frac{n-m}{T_{M}}\right)\right] \\
= & \frac{2 N_{y} \tau_{p} B_{e q}\left(f_{1}, f_{2}\right)}{B} \mathrm{e}^{-j 2 \pi\left(f_{1}-f_{2}\right) \tau_{\text {avg }}} \mathrm{e}^{j \pi \tau_{p}\left(f_{1}^{2}-f_{2}^{2}\right) / B} \mathrm{e}^{-j 2 \pi \tau_{p}\left(f_{1}-f_{2}\right) f_{c}\left(f_{1}, f_{2}\right) / B} \\
\times & \sum_{n=-N_{W}}^{N_{W}} \sum_{m=-N_{W}}^{N_{W}} W_{p}[n] W_{p}^{*}[m] \mathrm{e}^{j 2 \pi(n-m) \tau_{\text {vvg }} / T_{M}} \mathrm{e}^{j \pi \tau_{p}\left(n^{2}-m^{2}\right) /\left(B T_{M}^{2}\right)} \\
\times & \mathrm{e}^{-j 2 \pi \tau_{p}\left(n f_{1}-m f_{2}\right) /\left(B T_{M}\right)} \mathrm{e}^{j 2 \pi \tau_{p}(n-m) f_{c}\left(f_{1}, f_{2}\right) /\left(B T_{M}\right)} \\
\times & \operatorname{sinc}\left[B_{e q}\left(f_{1}, f_{2}\right) \frac{\tau_{p}}{B}\left(f_{1}-f_{2}-\frac{n-m}{T_{M}}\right)\right] .
\end{aligned}
$$

Analysis of Equation (70) reveals two sufficient conditions for WSS $Y_{C M}(f)$. Firstly, $B_{\text {eq }}\left(f_{1}, f_{2}\right)$ is either constant or a function of only $f_{1}-f_{2}$. Secondly, $f_{c}\left(f_{1}, f_{2}\right)=\left(f_{1}+f_{2}\right) / 2$.

\section{Simulation Results}

To demonstrate the correctness and utility of Equation (70), we simulate a radar having the parameter values listed in Table 1 . With these parameters a $1-\mathrm{kHz}$ frequency separation in the Fourier transform's output maps to a $1.5-\mathrm{m}$ slantrange separation.

To achieve various compromises between Rayleigh range resolution [2] and peak sidelobe levels [14], the radar can use the CT rectangular, Hamming, Blackman, and Kaiser windows, mathematically described by [15]

$$
\begin{gathered}
w_{R}(t)=\Pi\left[\left(t-\tau_{\text {avg }}\right) / T_{M}\right], \\
w_{H}(t)=\left\{0.54+0.46 \cos \left[2 \pi\left(1 / T_{M}\right)\left(t-\tau_{\text {avg }}\right)\right]\right\} \Pi\left[\left(t-\tau_{\text {avg }}\right) / T_{M}\right], \\
w_{B}(t)=\left\{0.42+0.5 \cos \left[2 \pi\left(1 / T_{M}\right)\left(t-\tau_{\text {avg }}\right)\right]\right. \\
\left.+0.08 \cos \left[2 \pi\left(2 / T_{M}\right)\left(t-\tau_{\text {avg }}\right)\right]\right\} \Pi\left[\left(t-\tau_{\text {avg }}\right) / T_{M}\right],
\end{gathered}
$$

and 
Table 1. Parameters of simulated radar system.

\begin{tabular}{cc}
\hline Parameter & Value \\
\hline$B$ & $10 \mathrm{MHz}$ \\
$\tau_{p}$ & $100 \mu \mathrm{s}$ \\
$R_{\min }$ & $19.5 \mathrm{~km}$ \\
$R_{\max }$ & $25.5 \mathrm{~km}$ \\
$\tau_{\min }=2 R_{\min } / c$ & $130 \mu \mathrm{s}$ \\
$\tau_{\max }=2 R_{\max } / c$ & $170 \mu \mathrm{s}$ \\
$\tau_{\text {avg }}=\left(\tau_{\min }+\tau_{\max }\right) / 2$ & $150 \mu \mathrm{s}$ \\
$f\left(R_{\min }\right)=B\left(\tau_{\text {avg }}-\tau_{\min }\right) / \tau_{p}$ & $2 \mathrm{MHz}$ \\
$f\left(R_{\max }\right)=B\left(\tau_{\text {avg }}-\tau_{\max }\right) / \tau_{p}$ & $-2 \mathrm{MHz}$ \\
$T_{M}=\tau_{\max }-\tau_{\min }+\tau_{p}$ & $140 \mu \mathrm{s}$ \\
$B_{M}=B T_{M} / \tau_{p}$ & $14 \mathrm{MHz}$ \\
\hline
\end{tabular}

$$
w_{K}(t)=\frac{I_{0}\left(\beta \sqrt{1-\left[2\left(t-\tau_{\text {avg }}\right) / T_{M}\right]^{2}}\right)}{I_{0}(\beta)} \Pi\left[\left(t-\tau_{\text {avg }}\right) / T_{M}\right],
$$

respectively. In Equation (74) $I_{0}$ is the zeroth-order modified Bessel function of the first kind with shaping parameter $\beta \geq 0$. We choose

$$
\beta=4
$$

to specify a Kaiser window having a temporally broader characteristic than the Hamming and Blackman windows, as shown in Figure 4.

Table 2 shows the key performance characteristics corresponding to these four windows, assuming the returned pulse is temporally centered in each window.

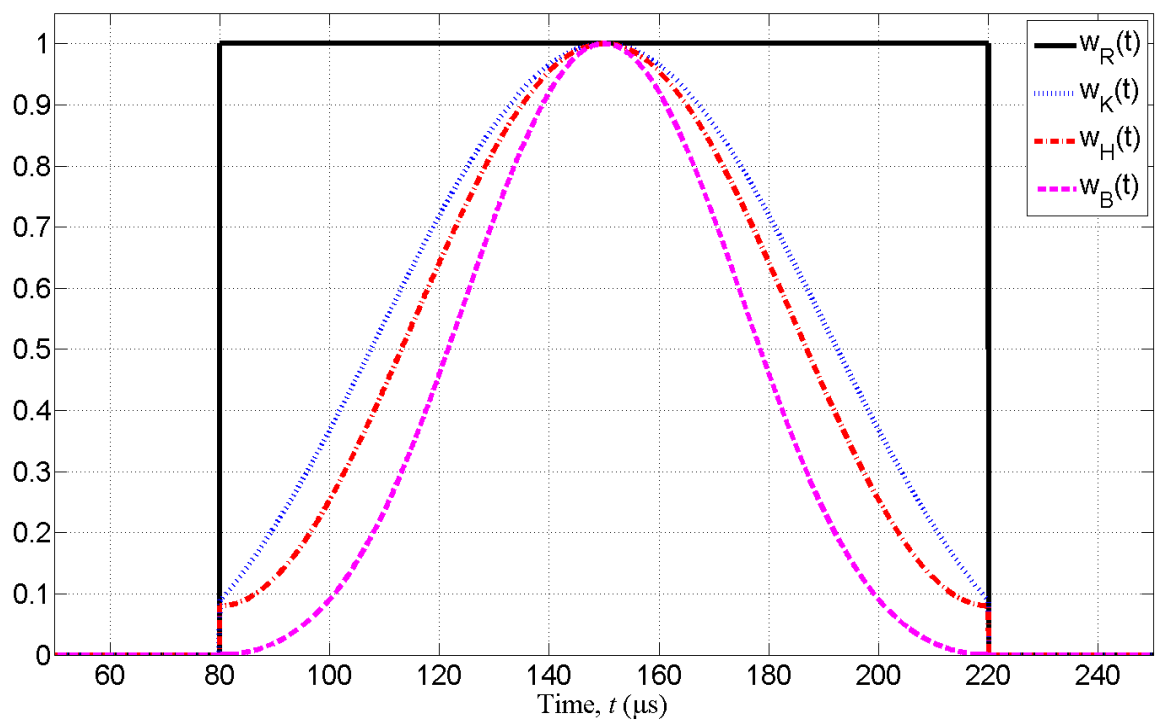

Figure 4. Windows used by simulated radar system. 
Table 2. Characteristics of available windows.

\begin{tabular}{ccc}
\hline Window & $\begin{array}{c}\text { Rayleigh Range } \\
\text { Resolution }(\mathrm{m})\end{array}$ & $\begin{array}{c}\text { Maximum Sidelobe Level below Peak } \\
\text { Response }(\mathrm{dB})\end{array}$ \\
\hline Rectangular & 15.0 & 13.3 \\
Hamming & 20.2 & 23.0 \\
Blackman & 26.4 & 35.7 \\
Kaiser $(\beta=4)$ & 18.6 & 20.0 \\
\hline
\end{tabular}

For convenience of simulation, we set $N_{y}$ to $1 \mathrm{~V}^{2} / \mathrm{Hz}$. We pass complex white, Gaussian noise with independent, equal-variance real and imaginary parts through a fifth-order Butterworth lowpass filter with bandwidth $B_{y} / 2$ and then spectrally translate the output noise by $f_{y}-f_{R F}$ to obtain complex noise with a PSD closely approximating Equation (41). For each considered case, 10,000 Monte-Carlo runs produce the data used to simulate the PDFs (through histograms) and the autocorrelation functions (through sample averages). We simulate two types of BLGN having practical significance.

\subsection{Case 1: Wideband Noise}

For this case we set

$$
f_{y}=f_{R F} \text {, }
$$

and we choose

$$
B_{y}=20 \mathrm{MHz}>B_{M}-2 f\left(R_{\max }\right)=18 \mathrm{MHz}
$$

to guarantee the BLGN's PSD always fully fills the complex stretch processor's "passband," i.e., the interval $\left[f\left(R_{\max }\right), f\left(R_{\min }\right)\right]$. This noise could represent internal receiver thermal noise or external broadband-noise jamming. Using Equation (63) and Equation (64), we determine that the BLGN corrupts the complex stretch processor's output from $f_{a}=-2 \mathrm{MHz}$ to $f_{b}=2 \mathrm{MHz}$ (i.e., all output frequencies of interest to this complex stretch processor). Thus, we will only consider values of $f_{1}$ and $f_{2}$ on $[-2 \mathrm{MHz}, 2 \mathrm{MHz}]$. Equation (65) and Equation (66) then respectively give

$$
f_{l}\left(f_{1}, f_{2}\right)=\max \left(f_{1}, f_{2}\right)-7 \mathrm{MHz}
$$

and

$$
f_{u}\left(f_{1}, f_{2}\right)=\min \left(f_{1}, f_{2}\right)+7 \mathrm{MHz} .
$$

Substituting Equation (78) and Equation (79) into Equation (68) and Equation (69) respectively gives

$$
f_{c}\left(f_{1}, f_{2}\right)=\left(f_{1}+f_{2}\right) / 2
$$

and

$$
B_{\text {eq }}\left(f_{1}, f_{2}\right)=14 \mathrm{MHz}-\left|f_{1}-f_{2}\right| .
$$

Clearly, Equation (80) and Equation (81) satisfy the sufficient condition for 
$Y_{C M}(f)$ being WSS. By substituting Equation (80) and Equation (81) into Equation (70), we can approximate the autocorrelation function as

$$
\begin{aligned}
& R_{Y_{C M}}\left(f_{1}, f_{2}\right) \approx 2 \times 10^{-11}\left(14 \times 10^{6}-\left|f_{1}-f_{2}\right|\right) \mathrm{e}^{-j 2 \pi\left(f_{1}-f_{2}\right)\left(1.5 \times 10^{-4}\right)} \\
& \times \sum_{n=-N_{W}}^{N_{W}} \sum_{m=-N_{W}}^{N_{W}} W_{p}[n] W_{p}^{*}[m] \mathrm{e}^{j 2 \pi(n-m)(15 / 14)} \mathrm{e}^{j \pi\left(n^{2}-m^{2}\right) / 1960} \mathrm{e}^{-j \pi(n+m)\left(f_{1}-f_{2}\right) /\left(14 \times 10^{6}\right)} \\
& \times \operatorname{sinc}\left\{\left(14 \times 10^{6}-\left|f_{1}-f_{2}\right|\right)\left(10^{-11}\right)\left[f_{1}-f_{2}-(n-m) /\left(1.4 \times 10^{-4}\right)\right]\right\} \\
& =R_{Y_{C M}}\left(f_{1}-f_{2}\right) .
\end{aligned}
$$

As expected, Equation (82) depends on only $f_{1}-f_{2}$, so the output noise is WSS for this case, regardless of the specific window employed.

\subsubsection{Case 1a: Rectangular Window}

By substituting Equation (71) into Equation (28), we obtain

$$
W_{p}[n]=\left\{\begin{array}{cc}
1, & n=0 \\
0, & \text { otherwise }
\end{array}\right.
$$

as the exact Fourier coefficients necessary to evaluate Equation (82). After significant simplification we obtain

$$
\begin{aligned}
R_{Y_{C M}}\left(f_{1}-f_{2}\right) \approx & 2 \times 10^{-11}\left(14 \times 10^{6}-\left|f_{1}-f_{2}\right|\right) \mathrm{e}^{-j 2 \pi\left(f_{1}-f_{2}\right)\left(1.5 \times 10^{-4}\right)} \\
& \times \operatorname{sinc}\left[\left(14 \times 10^{6}-\left|f_{1}-f_{2}\right|\right) 10^{-11}\left(f_{1}-f_{2}\right)\right]
\end{aligned}
$$

as the final expression for the output's theoretical autocorrelation function. In agreement with [3] [4], for any frequency considered by the complex stretch processor, the output noise will have a variance of

$$
R_{Y_{C M}}(0) \approx \frac{2 N_{y} \tau_{p} B_{e q}\left(f_{1}, f_{1}\right)}{B}=\frac{2 N_{y} \tau_{p} B_{M}}{B}=2 N_{y} T_{M}=2.8 \times 10^{-4} \mathrm{~V}^{2} / \mathrm{Hz}^{2} .
$$

Figure 5 shows overlays of the theoretical and numerically approximated
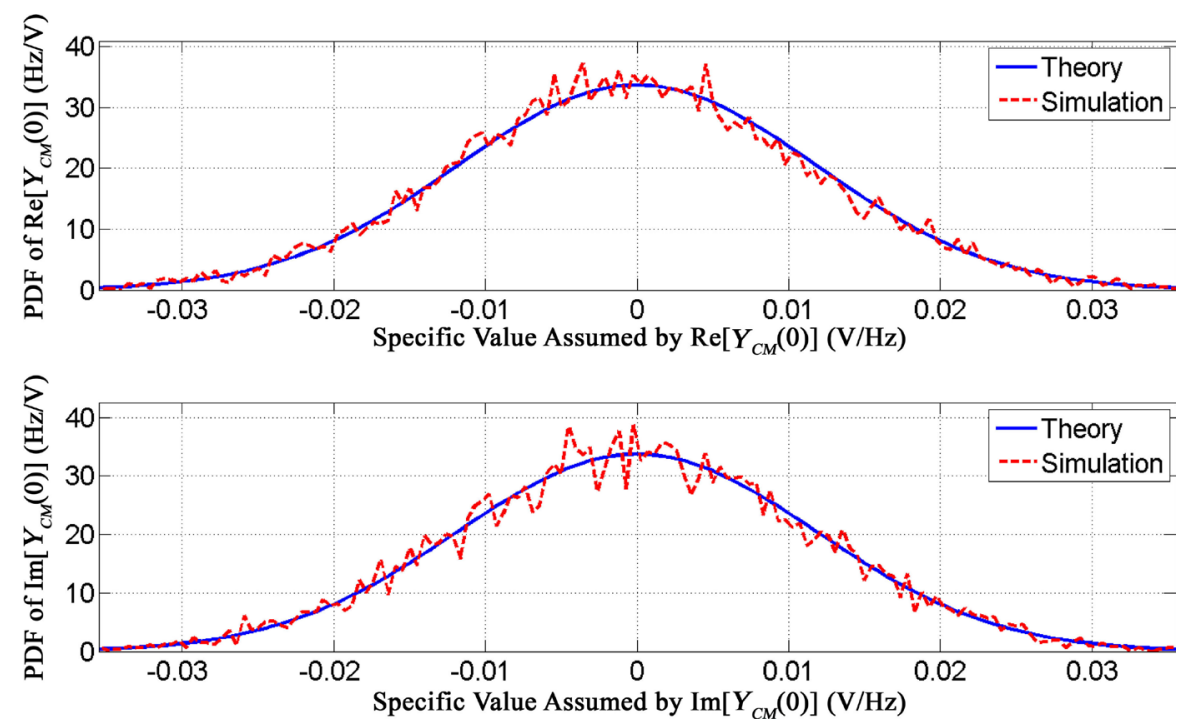

Figure 5. Theoretical and simulated PDFs for Case 1a. 
PDFs of the real and imaginary components of the complex stretch processor's output at $f_{1}-f_{2}=0$. The theoretical PDFs are Gaussian with mean zero and variance $0.5\left(2.8 \times 10^{-4} \mathrm{~V}^{2} / \mathrm{Hz}^{2}\right)=1.4 \times 10^{-4} \mathrm{~V}^{2} / \mathrm{Hz}^{2}$ (since we expect the real and imaginary components to each have half the total noise variance). Clearly, the simulated output's real and imaginary components both closely follow a Gaussian characteristic having the theoretically predicted mean and variance. The numerically approximated correlation coefficient for the simulated output's real and imaginary components is -0.00073 . Since this value is practically zero, the real and imaginary components are practically uncorrelated. Since the real and imaginary components are also Gaussian, they are practically independent, as previously stated.

Figure 6 shows excellent agreement between the theoretical and simulated autocorrelation functions. We conventionally consider output-noise components separated in frequency by a minimum of about $8.62 \mathrm{kHz}$ (the $3-\mathrm{dB}$ width of the main lobe of the autocorrelation function's magnitude) to be practically uncorrelated. The $8.62-\mathrm{kHz}$ frequency difference maps to a slant-range separation of $12.93 \mathrm{~m}$ which is below this radar's Rayleigh range resolution of $15 \mathrm{~m}$ (10 $\mathrm{kHz})$. Thus, if the radar samples the stretch processor's output every $15 \mathrm{~m}$ (10 $\mathrm{kHz}$ ), the BLGN-related components should be practically uncorrelated from one range sample to the next.

The complex correlation coefficient [16]

$$
\begin{aligned}
& \rho(f+\Delta f, f) \\
& \triangleq \frac{\operatorname{cov}\left[Y_{C M}(f+\Delta f) Y_{C M}^{*}(f)\right]}{\sqrt{\operatorname{var}\left[Y_{C M}(f+\Delta f)\right]} \sqrt{\operatorname{var}\left[Y_{C M}^{*}(f)\right]}} \\
& =\frac{E\left\{\left[Y_{C M}(f+\Delta f)-E\left[Y_{C M}(f+\Delta f)\right]\right]\left[Y_{C M}(f)-E\left[Y_{C M}(f)\right]\right]^{*}\right\}}{\sqrt{\operatorname{var}\left[Y_{C M}(f+\Delta f)\right]} \sqrt{\operatorname{var}\left[Y_{C M}(f)\right]}} \\
& =\frac{E\left[Y_{C M}(f+\Delta f) Y_{C M}^{*}(f)\right]}{\sqrt{\operatorname{var}\left[Y_{C M}(f+\Delta f)\right]} \sqrt{\operatorname{var}\left[Y_{C M}(f)\right]}} \\
& =\frac{R_{Y_{C M}}(f+\Delta f, f)}{\sqrt{R_{Y_{C M}}(f+\Delta f, f+\Delta f)} \sqrt{R_{Y_{C M}}(f, f)}}
\end{aligned}
$$

quantitatively characterizes the correlation between samples of $Y_{C M}(f)$ at frequencies $f+\Delta f$ and $f$. Since $Y_{C M}(f)$ is WSS for this case,

$$
\rho(f+\Delta f, f)=\frac{R_{Y_{C M}}(\Delta f)}{\sqrt{R_{Y_{C M}}(0)} \sqrt{R_{Y_{C M}}(0)}}=\frac{R_{Y_{C M}}(\Delta f)}{R_{Y_{C M}}(0)}=\rho(\Delta f) .
$$

We evaluate Equation (84) at $\Delta f=10 \mathrm{kHz}$ and $f=0$ and substitute the results into Equation (87) to obtain

$$
\rho(10 \mathrm{kHz})=\frac{R_{Y_{C M}}(10 \mathrm{kHz})}{R_{Y_{C M}}(0)} \approx 0.2160+j 0.0000 .
$$

Since $|\rho(10 \mathrm{kHz})|$ is approximately 0.2160 , the two samples of $Y_{C M}(f)$ are only slightly correlated. 

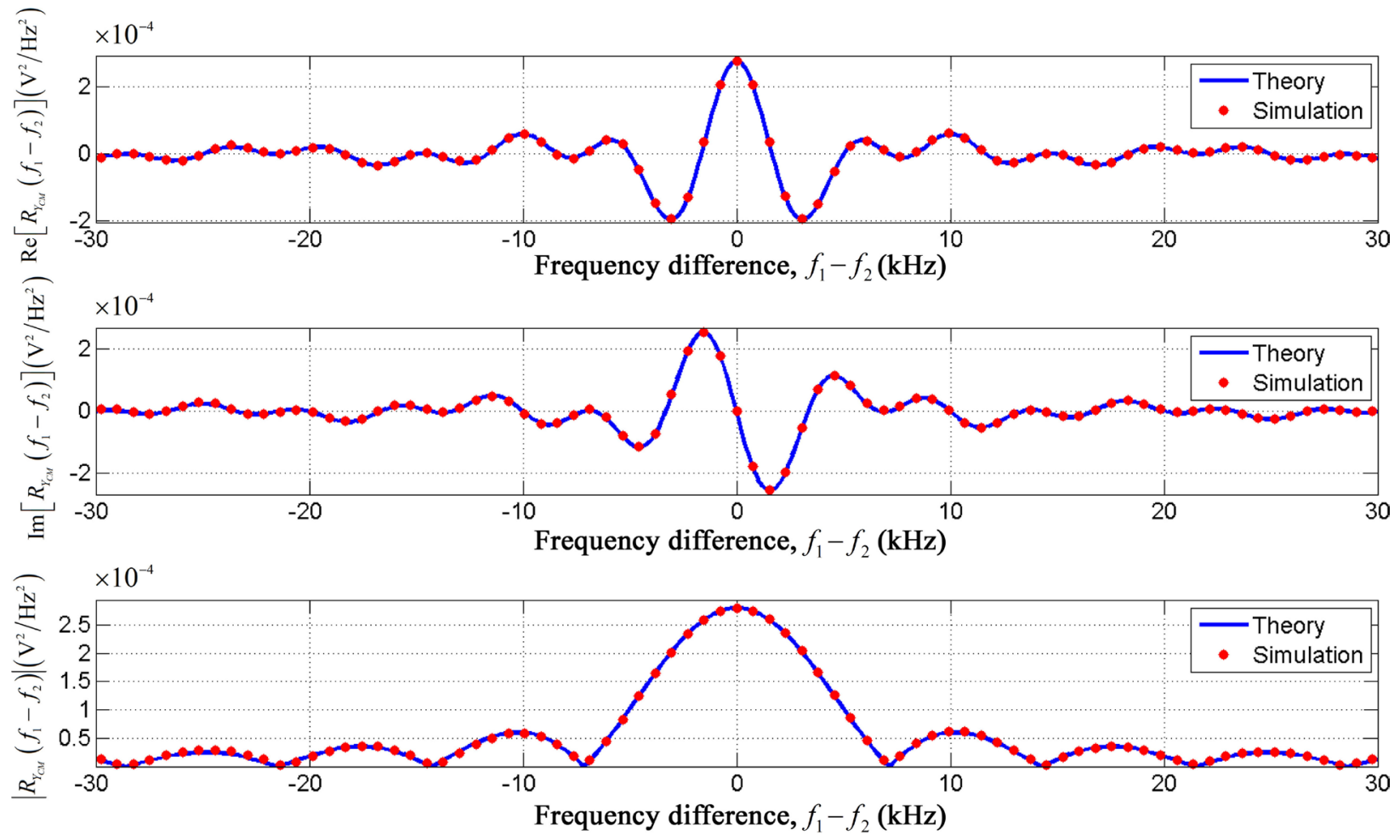

Figure 6. Theoretical and simulated autocorrelation functions for Case 1a.

\subsubsection{Case 1b: Hamming Window}

By substituting Equation (72) into Equation (28), we obtain

$$
W_{p}[n]= \begin{cases}0.23 \mathrm{e}^{j 2 \pi(15 / 14)}, & n=-1 \\ 0.54, & n=0 \\ 0.23 \mathrm{e}^{-j 2 \pi(15 / 14)}, & n=1 \\ 0, & \text { otherwise }\end{cases}
$$

as the exact Fourier coefficients necessary to evaluate Equation (82) in closed form. After significant simplification we obtain

$$
\begin{aligned}
R_{Y_{C M}}\left(f_{1}-f_{2}\right) \approx 2 \times 10^{-11}\left(14 \times 10^{6}-\left|f_{1}-f_{2}\right|\right) \mathrm{e}^{-j 2 \pi\left(f_{1}-f_{2}\right)\left(1.5 \times 10^{-4}\right)} \\
\times\left\{\left\{0.54^{2}+2\left(0.23^{2}\right) \cos \left[2 \pi\left(f_{1}-f_{2}\right) / 14 \times 10^{6}\right]\right\}\right. \\
\quad \times \operatorname{sinc}\left[\left(14 \times 10^{6}-\left|f_{1}-f_{2}\right|\right) 10^{-11}\left(f_{1}-f_{2}\right)\right] \\
+2(0.54)(0.23)\left\{\cos \left[\pi\left(f_{1}-f_{2}\right) / 14 \times 10^{6}-\pi / 1960\right]\right. \\
\times \operatorname{sinc}\left[\left(14 \times 10^{6}-\left|f_{1}-f_{2}\right|\right) 10^{-11}\left(f_{1}-f_{2}-1 / 1.4 \times 10^{-4}\right)\right] \\
+\cos \left[\pi\left(f_{1}-f_{2}\right) / 14 \times 10^{6}+\pi / 1960\right] \\
\left.\times \operatorname{sinc}\left[\left(14 \times 10^{6}-\left|f_{1}-f_{2}\right|\right) 10^{-11}\left(f_{1}-f_{2}+1 / 1.4 \times 10^{-4}\right)\right]\right\} \\
+\left(0.23^{2}\right)\left\{\operatorname{sinc}\left[\left(14 \times 10^{6}-\left|f_{1}-f_{2}\right|\right) 10^{-11}\left(f_{1}-f_{2}-2 / 1.4 \times 10^{-4}\right)\right]\right. \\
\left.\left.+\operatorname{sinc}\left[\left(14 \times 10^{6}-\left|f_{1}-f_{2}\right|\right) 10^{-11}\left(f_{1}-f_{2}+2 / 1.4 \times 10^{-4}\right)\right]\right\}\right\}
\end{aligned}
$$


as the final closed-form expression for the output's theoretical autocorrelation function. For any frequency considered by the complex stretch processor, the output noise will have a variance of about

$$
R_{Y_{C M}}(0) \approx 1.113 \times 10^{-4} \mathrm{~V}^{2} / \mathrm{Hz}^{2} .
$$

Figure 7 shows overlays of the theoretical and numerically approximated PDFs of the real and imaginary components of the complex stretch processor's output at $f_{1}-f_{2}=0$. The theoretical PDFs are Gaussian with mean zero and variance $0.5\left(1.113 \times 10^{-4} \mathrm{~V}^{2} / \mathrm{Hz}^{2}\right)=5.56 \times 10^{-5} \mathrm{~V}^{2} / \mathrm{Hz}^{2}$. Clearly, the simulated output's real and imaginary components both closely follow a Gaussian characteristic having the theoretically predicted mean and variance. The numerically approximated correlation coefficient for the simulated output's real and imaginary components is 0.0047 , indicating the two Gaussian components are practically independent.

Figure 8 shows excellent agreement between the theoretical and simulated autocorrelation functions. Using the previously stated convention, we consider output noise components separated in frequency by a minimum of about 17.3 $\mathrm{kHz}$ to be practically uncorrelated. This frequency difference maps to a slantrange separation of $25.9 \mathrm{~m}$ which exceeds this radar's Rayleigh range resolution of $20.2 \mathrm{~m}$ by about $28 \%$. If the radar samples the stretch processor's output every $20.2 \mathrm{~m}(13.47 \mathrm{kHz})$, the BLGN-related components in any two adjacent range samples will have a complex correlation coefficient of

$$
\rho(13.467 \mathrm{kHz})=R_{\mathrm{Y}_{C M}}(13.467 \mathrm{kHz}) / R_{Y_{C M}}(0) \approx 0.1685-j 0.0209 .
$$

Since $|\rho(13.467 \mathrm{kHz})|$ is approximately 0.1698 , the two samples of $Y_{C M}(f)$ are only slightly correlated despite the radar's range-sampling interval being somewhat less in extent than the conventionally defined range-decorrelation interval.
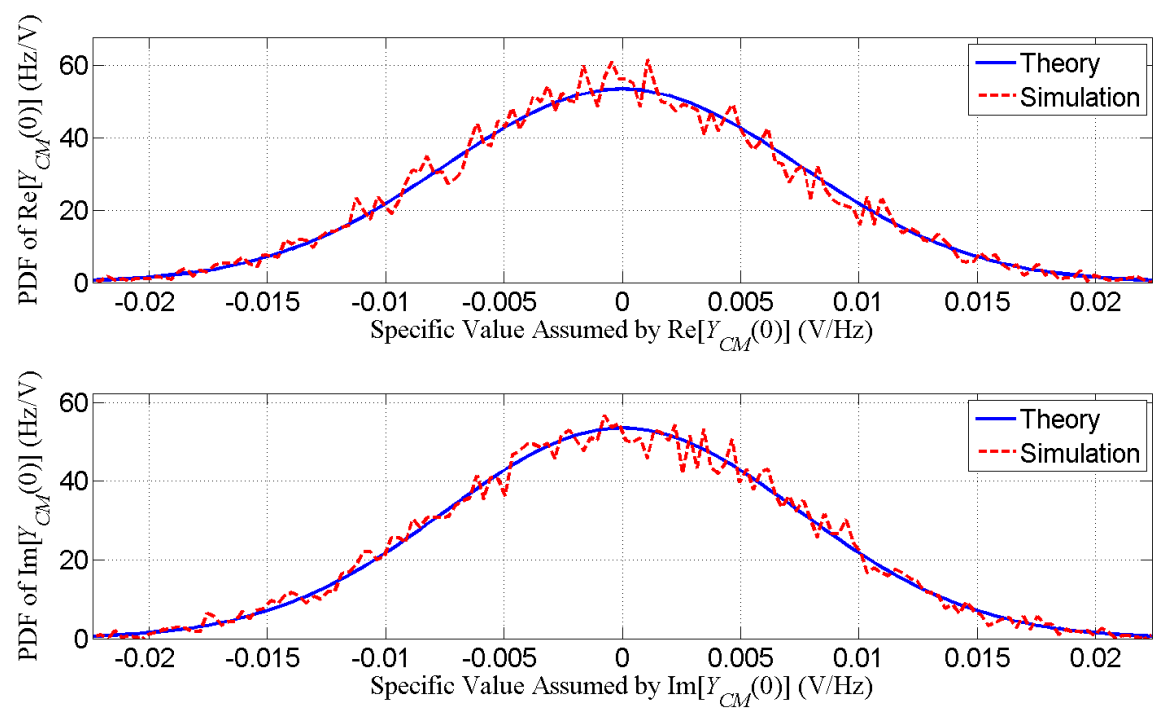

Figure 7. Theoretical and simulated PDFs for Case $1 \mathrm{~b}$. 

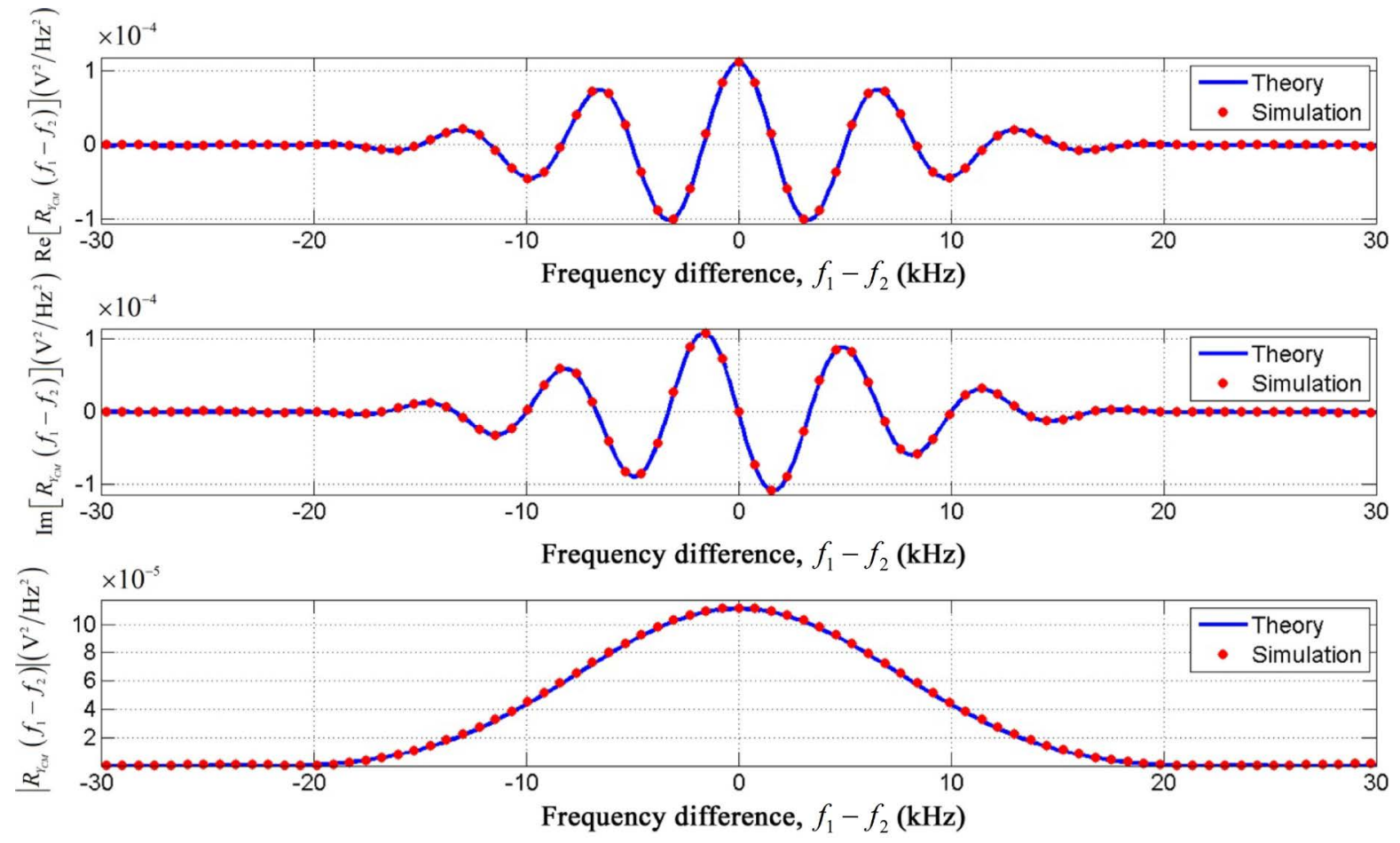

Figure 8. Theoretical and simulated autocorrelation functions for Case $1 \mathrm{~b}$.

\subsubsection{Case 1c: Blackman Window}

By substituting Equation (73) into Equation (28), we obtain

$$
W_{p}[n]= \begin{cases}0.04 \mathrm{e}^{j 2 \pi(15 / 7)}, & n=-2 \\ 0.25 \mathrm{e}^{j 2 \pi(15 / 14)}, & n=-1 \\ 0.42, & n=0 \\ 0.25 \mathrm{e}^{-j 2 \pi(15 / 14)}, & n=1 \\ 0.04 \mathrm{e}^{-j 2 \pi(15 / 7)}, & n=2 \\ 0, & \text { otherwise }\end{cases}
$$

as the exact Fourier coefficients necessary to evaluate Equation (82) in closed form. Note: For $N_{W}=2$, the double summation in Equation (82) produces $\left(2 N_{W}+1\right)^{2}=25$ terms; even after significant simplification, the closed-form expression for the theoretical autocorrelation function is relatively unwieldy, so we omit it. For any frequency considered by the complex stretch processor, the complex output noise will have a variance of about

$$
R_{Y_{C M}}(0) \approx 8.529 \times 10^{-5} \mathrm{~V}^{2} / \mathrm{Hz}^{2} \text {. }
$$

Figure 9 shows overlays of the theoretical PDFs and the numerically approximated PDFs of the real and imaginary components of the simulated complex stretch processor's output at $f_{1}-f_{2}=0$. The theoretical PDFs are Gaussian with mean zero and variance $0.5\left(8.529 \times 10^{-5} \mathrm{~V}^{2} / \mathrm{Hz}^{2}\right)=4.264 \times 10^{-5} \mathrm{~V}^{2} / \mathrm{Hz}^{2}$. Clearly, the simulated output's real and imaginary components both closely follow a Gaussian characteristic having the theoretically predicted mean and variance. The numerically approximated correlation coefficient for the simulated output's real and imaginary components is 0.0085 , indicating the two Gaussian 

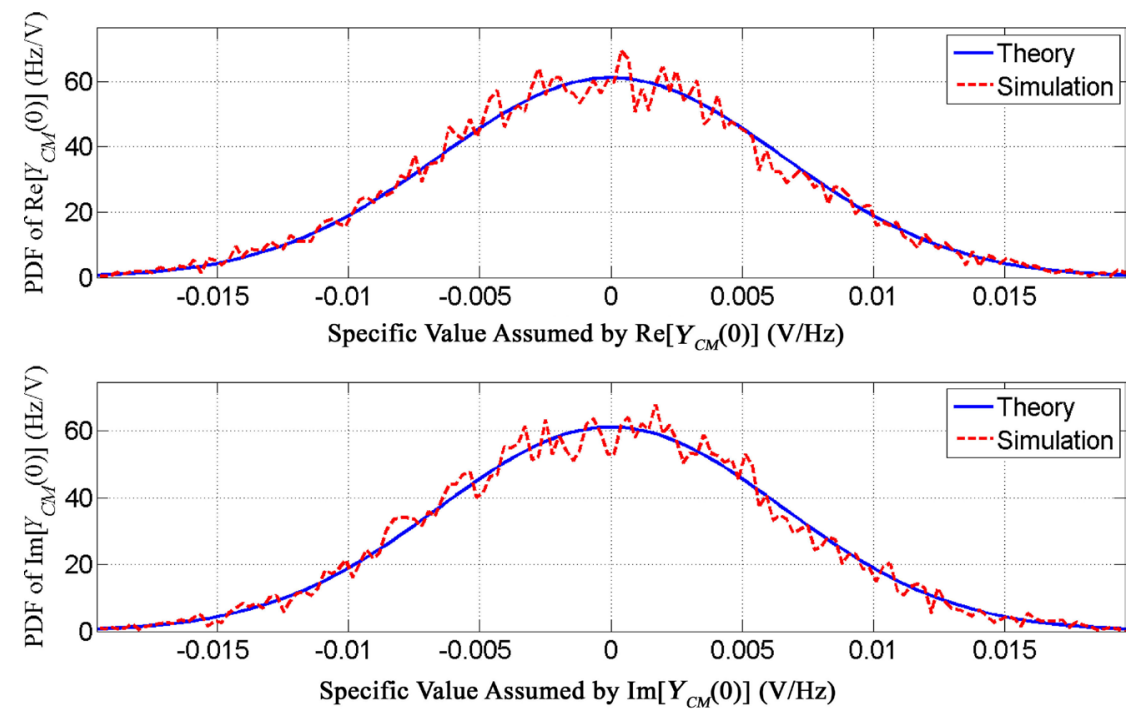

Figure 9. Theoretical and simulated PDFs for Case 1c.

components are practically independent.

Figure 10 shows excellent agreement between the theoretical and simulated autocorrelation functions. Using the previously specified convention, we consider output noise components separated in frequency by a minimum of about $22.3 \mathrm{kHz}$ to be practically uncorrelated. This frequency difference maps to a slant-range separation of $33.5 \mathrm{~m}$ which exceeds this radar's Rayleigh range resolution of $26.4 \mathrm{~m}$ by about $27 \%$. If the radar samples the stretch processor's output every $26.4 \mathrm{~m}(17.6 \mathrm{kHz})$, the BLGN-related components in any two adjacent
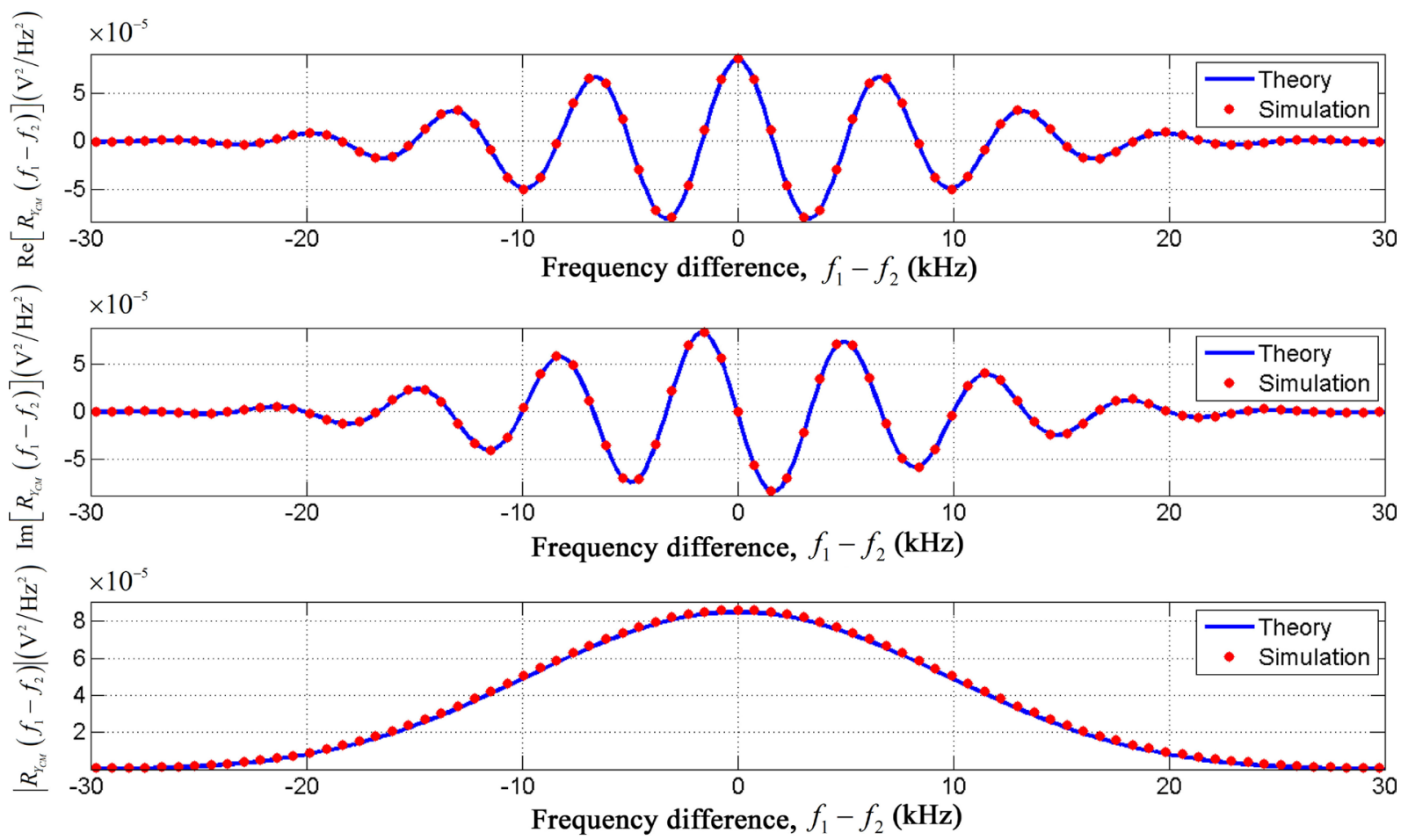

Figure 10. Theoretical and simulated autocorrelation functions for Case 1c. 
range samples will have a complex correlation coefficient of

$$
\rho(17.600 \mathrm{kHz})=R_{Y_{C M}}(17.600 \mathrm{kHz}) / R_{Y_{C M}}(0) \approx-0.1070+j 0.1293 .
$$

Since $|\rho(17.6 \mathrm{kHz})|$ is approximately 0.1678 , the two samples of $Y_{C M}(f)$ are only slightly correlated despite the radar's range-sampling interval being somewhat less in extent than the conventionally defined range-decorrelation interval.

\subsection{Case 2: Narrowband Interference with Kaiser Window}

We next consider BLGN with center frequency $f_{y}=f_{R F}+3 \mathrm{MHz}$ and bandwidth $B_{y}=2 \mathrm{MHz}$. This BLGN might represent a relatively narrowband interference source emitting in the radar's transmit band. Using Equation (63) and Equation (64), we determine the BLGN corrupts the complex stretch processor's output from $f_{a}=-2 \mathrm{MHz}$ to $f_{b}=2 \mathrm{MHz}$. Thus, we will only consider values of $f_{1}$ and $f_{2}$ on $[-2 \mathrm{MHz}, 2 \mathrm{MHz}]$. Equation (65) and Equation (66) then respectively give

$$
f_{l}\left(f_{1}, f_{2}\right)=2 \mathrm{MHz}
$$

and

$$
f_{u}\left(f_{1}, f_{2}\right)=4 \mathrm{MHz}
$$

For this case the stretch processor uses the Kaiser window specified by Equation (74) and Equation (75). The previously considered rectangular, Hamming, and Blackman windows have only a small number of nonzero Fourier coefficients, the exact values of which can be obtained from a straightforward application of Equation (28). In contrast any Kaiser window with $\beta>0$ generally has an infinite number of nonzero Fourier coefficients which can only be approximated (with arbitrary accuracy using numerical integration) by substituting Equation (74) into Equation (28). Table 3 shows the Fourier coefficients, rounded to seven decimal places, for the specified Kaiser window and $|n| \leq 7$.

Substituting Equation (96) and Equation (97) into Equation (68) and Equation (69) respectively gives

$$
f_{c}\left(f_{1}, f_{2}\right)=3 \mathrm{MHz}
$$

and

$$
B_{e q}\left(f_{1}, f_{2}\right)=2 \mathrm{MHz} .
$$

Equation (98) does not satisfy the sufficient condition for $Y_{C M}(f)$ being WSS, so we do not expect $Y_{C M}(f)$ to be WSS. Substituting Equation (98) and Equation (99) into Equation (70), we approximate the theoretical autocorrelation function as

$$
\begin{aligned}
R_{Y_{C M}}\left(f_{1}, f_{2}\right) \approx & 4 \times 10^{-5} \mathrm{e}^{-j 2 \pi\left(f_{1}-f_{2}\right)\left(1.8 \times 10^{-4}\right)} \mathrm{e}^{j 10^{-11} \pi\left(f_{1}^{2}-f_{2}^{2}\right)} \\
& \times \sum_{n=-N_{W}}^{N_{W}} \sum_{m=-N_{W}}^{N_{W}} W_{p}[n] W_{p}^{*}[m] \mathrm{e}^{j 2 \pi(n-m)(15 / 14)} \mathrm{e}^{j \pi\left(n^{2}-m^{2}\right) / 1960} \\
& \times \mathrm{e}^{-j 2 \pi\left(n f_{1}-m f_{2}\right) / 14 \times 10^{6}} \mathrm{e}^{j 2 \pi(n-m)(3 / 14)} \\
& \times \operatorname{sinc}\left[2 \times 10^{-5}\left(f_{1}-f_{2}-\frac{n-m}{14 \times 10^{-5}}\right)\right] .
\end{aligned}
$$


Table 3. Significant Fourier coefficients of the specified Kaiser window.

\begin{tabular}{ccc}
\hline$n$ & $W_{p}[n]$ & $\left|W_{p}[n]\right|$ \\
\hline-7 & $-0.0014676-j 0.0000000$ & 0.0014676 \\
-6 & $0.0018014-j 0.0008675$ & 0.0019994 \\
-5 & $-0.0017977+j 0.0022542$ & 0.0028833 \\
-4 & $0.0010050-j 0.0044031$ & 0.0045163 \\
-3 & $0.0017942+j 0.0078608$ & 0.0080629 \\
-2 & $-0.0112846-j 0.0141504$ & 0.0180991 \\
-1 & $0.1901075+j 0.0915510$ & 0.2110034 \\
0 & 0.6036566 & 0.6036566 \\
1 & $0.1901075-j 0.0915520$ & 0.2110034 \\
2 & $-0.0112846+j 0.0141504$ & 0.0180991 \\
3 & $0.0017942-j 0.0078608$ & 0.0080629 \\
4 & $0.0010050+j 0.0044031$ & 0.0045163 \\
5 & $-0.0017977-j 0.0022542$ & 0.0028833 \\
6 & $0.0018014+j 0.0008675$ & 0.0019994 \\
7 & $-0.0014676+j 0.000000$ & 0.0014676 \\
\hline
\end{tabular}

Since Equation (100) does not depend on only $f_{1}-f_{2}$, the output noise is not WSS for this case, as expected. Equation (100) finds practical application in the simulation of narrowband interference competing with a target return appearing at a particular frequency of the Fourier transform's output. Specifically, suppose a target at slant range $R_{t}$ appears at corresponding frequency $f_{t}$ in the Fourier transform's output. Evaluating Equation (100) with variable $f$ assuming the role of $f_{1}$ and fixed value $f_{t}$ assuming the role of $f_{2}$ statistically characterizes the noise at any frequency $f$ near the target's frequency $f_{t}$. We consider two target ranges and assume $N_{W}=5$.

\subsubsection{Case 2a: Target Range of $20 \mathrm{~km}$}

Evaluating Equation (100) using the values in Table 2 and

$$
f_{1}=f_{2}=f_{t}=1666.26 \mathrm{kHz}
$$

gives the variance of the complex stretch processor's output noise at $R_{t}=20 \mathrm{~km}$ as

$$
R_{Y_{C M}}\left(f_{t}, f_{t}\right) \approx 3.4566 \times 10^{-5} \mathrm{~V}^{2} / \mathrm{Hz}^{2} .
$$

Figure 11 shows overlays of the theoretical PDFs and the numerically approximated PDFs of the real and imaginary components of the simulated complex stretch processor's output at $f_{1}=f_{2}=f_{t}=1666.26 \mathrm{kHz}$. The theoretical PDFs are Gaussian with mean zero and variance

$0.5\left(3.4566 \times 10^{-5} \mathrm{~V}^{2} / \mathrm{Hz}^{2}\right)=1.7283 \times 10^{-5} \mathrm{~V}^{2} / \mathrm{Hz}^{2}$. Clearly, the simulated output's real and imaginary components both closely follow a Gaussian characteristic having the theoretically predicted mean and variance. The numerically 

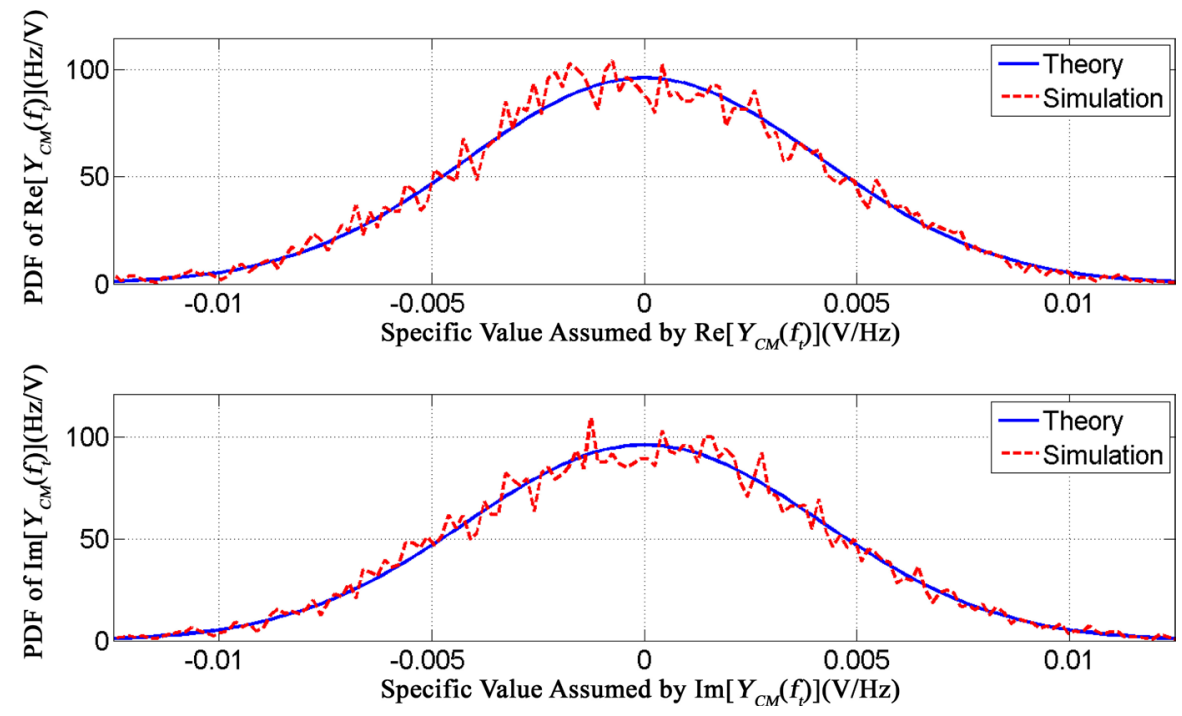

Figure 11. Theoretical and simulated PDFs for Case 2a.

approximated correlation coefficient for the simulated output's real and imaginary components is -0.0047 , indicating the two Gaussian components are practically independent.

Since we have assumed $N_{W}=5$, the double summation in Equation (100) initially produces $\left(2 N_{W}+1\right)^{2}=121$ terms, indicating that, even after significant simplification, the closed-form expression for Equation (100) will certainly be mathematically unwieldy. Therefore, we make no attempt to obtain a closedform solution without the double summation. Figure 12 shows excellent agree-
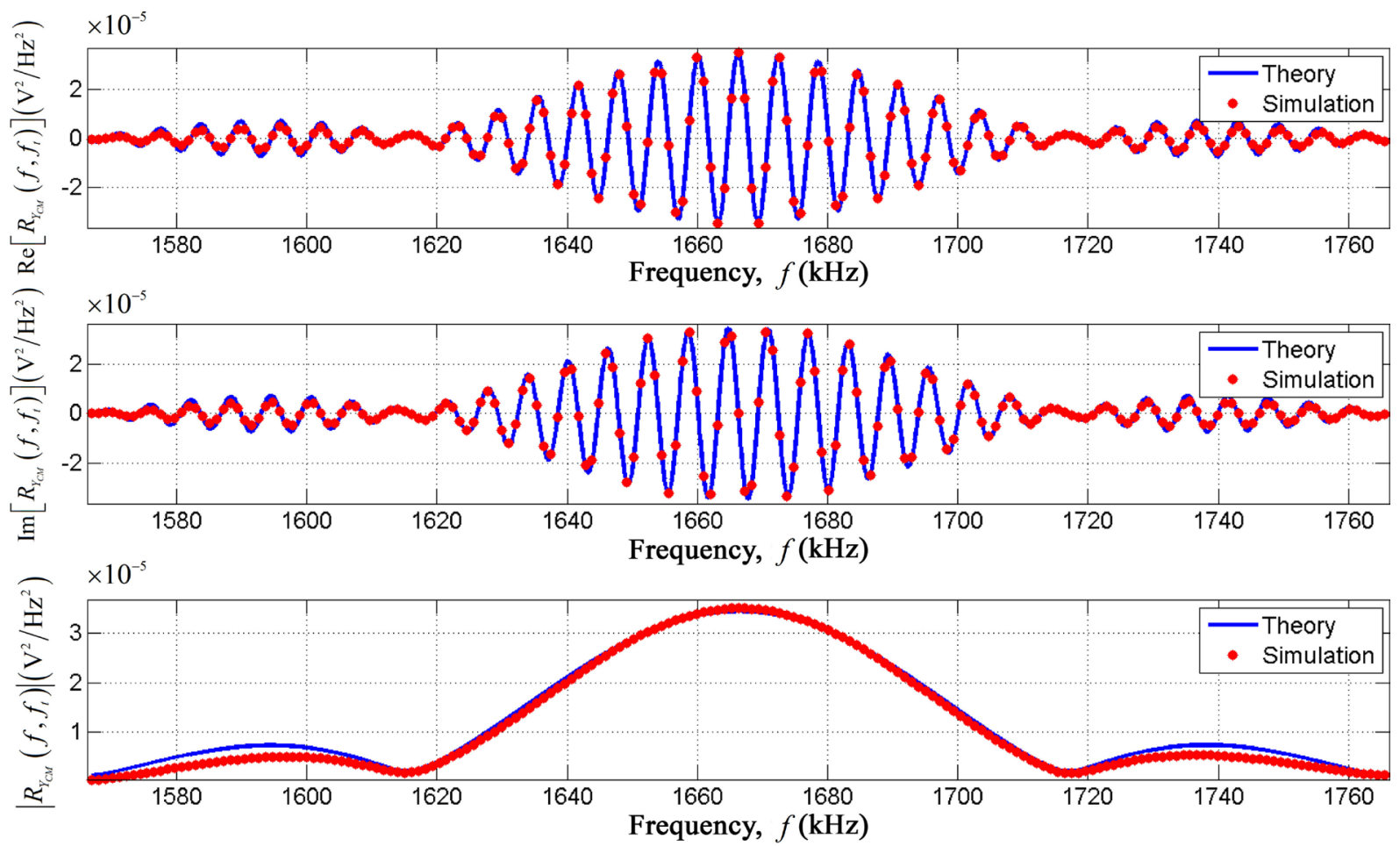

Figure 12. Theoretical and simulated autocorrelation functions for Case 2a. 
ment between the theoretical and simulated autocorrelation functions. Using the previously specified convention, we consider output noise components separated in frequency by a minimum of about $61.336 \mathrm{kHz}$ to be practically uncorrelated. This frequency difference maps to a slant-range separation of $92.0 \mathrm{~m}$ which greatly exceeds this radar's Rayleigh range resolution of $18.6 \mathrm{~m}$. If the radar samples the stretch processor's output every $18.6 \mathrm{~m}(12.4 \mathrm{kHz})$, the BLGNrelated components in any two adjacent range samples will have a complex correlation coefficient of

$$
\begin{aligned}
& \rho\left(f_{t}+12.4 \mathrm{kHz}, f_{t}\right) \\
& =\frac{R_{Y_{C M}}\left(f_{t}+12.4 \mathrm{kHz}, f_{t}\right)}{\sqrt{R_{Y_{C M}}\left(f_{t}+12.4 \mathrm{kHz}, f_{t}+12.4 \mathrm{kHz}\right)} \sqrt{R_{Y_{C M}}\left(f_{t}, f_{t}\right)}} \\
& \approx 0.899-j 0.094 .
\end{aligned}
$$

Since $\left|\rho\left(f_{t}+12.4 \mathrm{kHz}, f_{t}\right)\right|$ is approximately 0.9042 , the two samples of $Y_{C M}(f)$ are highly correlated-an expected result considering the conventional range-decorrelation interval's extent significantly exceeds the radar's rangesampling interval.

\subsubsection{Case $2 \mathrm{~b}$ : Target Range of $25 \mathrm{~km}$}

Evaluating Equation (100) using the values in Table 2 and

$$
f_{1}=f_{2}=f_{t}=-1666.26 \mathrm{kHz}
$$

gives the variance of the complex stretch processor's output noise at $R_{t}=25 \mathrm{~km}$ as

$$
R_{Y_{C M}}\left(f_{t}, f_{t}\right) \approx 7.7327 \times 10^{-6} \mathrm{~V}^{2} / \mathrm{Hz}^{2} .
$$

Figure 13 shows overlays of the theoretical PDFs and the numerically approximated PDFs of the real and imaginary components of the simulated complex stretch processor's output at $f_{1}=f_{2}=f_{t}=-1666.26 \mathrm{kHz}$. The theoretical
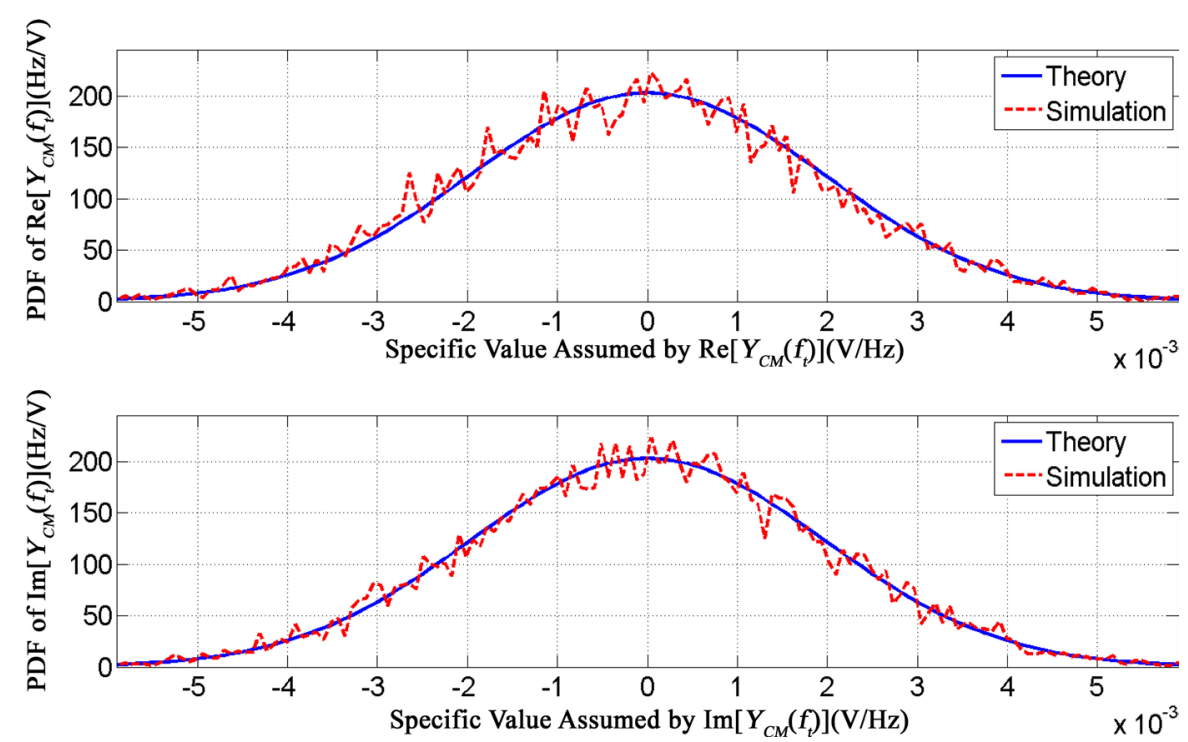

Figure 13. Theoretical and simulated PDFs for Case 2b. 
PDFs are Gaussian with mean zero and variance

$0.5\left(7.7327 \times 10^{-6} \mathrm{~V}^{2} / \mathrm{Hz}^{2}\right)=3.8644 \times 10^{-6} \mathrm{~V}^{2} / \mathrm{Hz}^{2}$. Clearly, the simulated output's real and imaginary components both closely follow a Gaussian characteristic having the theoretically predicted mean and variance. The numerically approximated correlation coefficient for the simulated output's real and imaginary components is 0.0018 , implying the two Gaussian components are practically independent.

Figure 14 shows excellent agreement between the theoretical and simulated autocorrelation functions. Using the previously specified convention, we consider output noise components separated in frequency by a minimum of about $68.2 \mathrm{kHz}$ to be practically uncorrelated. This frequency difference maps to a slant-range separation of $102.3 \mathrm{~m}$ which greatly exceeds this radar's Rayleigh range resolution of $18.6 \mathrm{~m}$. If the radar samples the stretch processor's output every $18.6 \mathrm{~m}(12.4 \mathrm{kHz})$, the BLGN-related components in any two adjacent range samples will have a complex correlation coefficient of

$$
\begin{aligned}
& \rho\left(f_{t}+12.4 \mathrm{kHz}, f_{t}\right) \\
& =\frac{R_{Y_{C M}}\left(f_{t}+12.4 \mathrm{kHz}, f_{t}\right)}{\sqrt{R_{Y_{C M}}\left(f_{t}+12.4 \mathrm{kHz}, f_{t}+12.4 \mathrm{kHz}\right)} \sqrt{R_{Y_{C M}}\left(f_{t}, f_{t}\right)}} \\
& \approx-0.7603-j 0.5119 .
\end{aligned}
$$

Since $\left|\rho\left(f_{t}+12.4 \mathrm{kHz}, f_{t}\right)\right|$ is approximately 0.9165 , the two samples of $Y_{C M}(f)$ are highly correlated-an expected result considering the conventional range-decorrelation interval's extent significantly exceeds the radar's rangesampling interval.
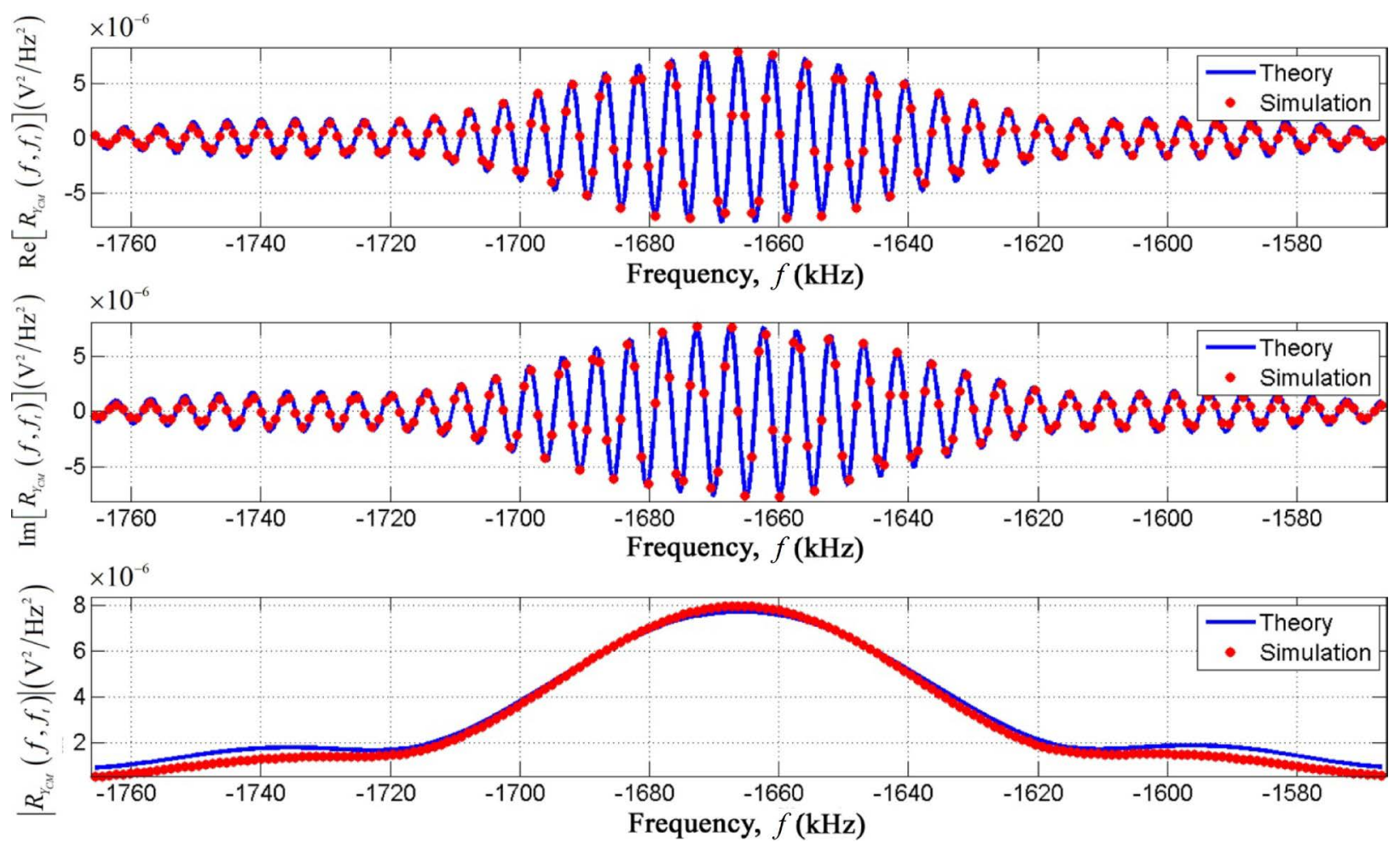

Figure 14. Theoretical and simulated autocorrelation functions for Case $2 \mathrm{~b}$. 


\section{Conclusion}

This paper presented a detailed mathematical development which characterized the response to arbitrarily bandlimited Gaussian noise of a complex stretch processor using a conventional range-sidelobe-reduction window. The paper specified the complex stretch processor's functional structure and the input BLGN's mathematical description. The subsequent development then propagated the BLGN through the complex stretch processor's functional components, characterizing the noise at the key components' outputs.

The effort produced four significant findings. Firstly, the final output is complex, zero-mean, Gaussian noise with equal variance in its independent real and imaginary components. Secondly, the output noise's autocorrelation function has a highly accurate closed-form approximation readily determined from the radar's and input BLGN's parameters. Thirdly, the output noise is generally not WSS (whereas it is for the case of a matched filter), which may complicate highfidelity modeling. Fourthly, we may determine the correlation between the noise components of any two output range samples by evaluating the complex correlation coefficient using the derived autocorrelation function.

The windows considered in this effort were all conventional (i.e., real, symmetric, and lowpass). The described approach also applies to complex and/or asymmetric windows so long as those functions have energy spectral densities concentrated around $f=0 \mathrm{~Hz}$. In addition the approach readily extends to unconventional windows having energy spectral densities concentrated around a nonzero frequency.

\section{References}

[1] Caputi, W. (1971) Stretch: A Time-Transformation Technique. IEEE Transactions on Aerospace and Electronic Systems, 7, 269-278. https://doi.org/10.1109/TAES.1971.310366

[2] Richards, M. (2005) Fundamentals of Radar Signal Processing. McGraw-Hill, New York.

[3] Budge, M. and German, S. (2015) Basic Radar Analysis. Artech House, Boston.

[4] Melvin, W. and Scheer, J. (2013) Principles of Modern Radar, Vol. II: Advanced Techniques. SciTech Publishing, Edison.

[5] Nathanson, F. (1999) Radar Design Principles. 2nd Edition, SciTech Publishing, Mendham.

[6] Mahafza, B. (2013) Radar Systems Analysis and Design using MATLAB. 3rd Edition, CRC Press, Boca Raton.

[7] Spitzmiller, J. (2017) The Complex Stretch Processor's Response to Arbitrarily Bandlimited Gaussian Noise. Proceedings of the 2017 IEEE SoutheastCon, Charlotte. https://doi.org/10.1109/SECON.2017.7925375

[8] Spitzmiller, J. (2017) The Response to Arbitrarily Bandlimited Gaussian Noise of the Complex Stretch Processor using Certain Range-Sidelobe-Reduction Windows. Proceedings of the 2017 IEEE National Aerospace \& Electronics Conference, Dayton. https://doi.org/10.1109/NAECON.2017.8268779

[9] Peebles, P. (1998) Radar Principles. John Wiley \& Sons, Inc., New York. 
[10] Cumming, I. and Wong, F. (2005) Digital Processing of Synthetic Aperture Radar Data. Artech House, Norwood.

[11] Peebles, P. (1993) Probability, Random Variables, and Random Signal Principles. 3rd Edition, McGraw-Hill, New York.

[12] Ziemer, R. and Tranter, W. (1995) Principles of Communications. 4th Edition, John Wiley \& Sons, Inc., New York.

[13] Papoulis, A. (1991) Probability, Random Variables, and Stochastic Processes. 3rd Edition, McGraw-Hill, Inc., New York.

[14] Oppenheim, A. and Schafer, R. (1989) Discrete-Time Signal Processing. Prentice-Hall, Inc., Englewood Cliffs.

[15] Stanley, W., Dougherty, G. and Dougherty, R. (1984) Digital Signal Processing. Reston Publishing Company, Inc., Reston.

[16] Urkowitz, H. (1983) Signal Theory and Random Processes. Artech House, Inc., Dedham. 